CERN-PH-EP/2007-005

16 March 2007

\title{
THE HARP RESISTIVE PLATE CHAMBERS: CHARACTERISTICS AND PHYSICS PERFORMANCE
}

\begin{abstract}
The HARP Resistive Plate Chamber (RPC) system was designed for time-of-flight measurement in the large-angle acceptance region of the HARP spectrometer. It comprised 46 four-gap glass RPCs covering an area of $\sim 8 \mathrm{~m}^{2}$. The design of the RPCs, their operation, intrinsic properties, and system performance are described. The intrinsic time resolution of the RPCs is better than 130 ps leading to a system time resolution of $\sim 175 \mathrm{ps}$.
\end{abstract}

I. Boyko, G. Chelkov, D. Dedovitch, A. Elagin, M. Gostkin, A. Guskov, Z. Kroumchtein, Yu. Nefedov, K. Nikolaev, A. Zhemchugov

Joint Institute for Nuclear Research, Dubna, Russia

R. Dumps, F. Dydak, L. Linssen, J. Wotschack

CERN, Geneva, Switzerland

V. Ammosov, V. Gapienko, V. Koreshev, A. Semak, Yu. Sviridov, E. Usenko, V. Zaets

Institute for High Energy Physics, Protvino, Russia

(Submitted to Nucl. Instrum. Methods Phys. Res. A) 


\section{INTRODUCTION}

The HARP experiment carried out a programme of systematic and precise measurements of hadron production by protons and pions with momenta from 1.5 to $15 \mathrm{GeV} / c$. The experiment was performed at the CERN Proton Synchrotron between May 2001 and October 2002 with a set of targets ranging from hydrogen to lead.

The main objective was to acquire adequate knowledge of pion yields for an optimal design of the proton driver of a neutrino factory, and to improve substantially the calculation of the atmospheric neutrino flux which is needed for a refined study of neutrino oscillations with atmospheric neutrinos. Further objectives were improving the input to Monte Carlo generators of secondary hadrons and better predictions of neutrino beam fluxes.

The HARP detector was designed to measure particle production over the full solid angle. It comprised a large-angle spectrometer and a forward spectrometer; the latter with a dipole magnet and drift chambers for momentum measurement, complemented by a threshold Cherenkov counter, a time-of-flight wall, and an electromagnetic calorimeter for particle identification. The large-angle spectrometer consisted of a Time Projection Chamber (TPC) embedded in a $0.7 \mathrm{~T}$ solenoidal magnet, and two arrays of Resistive Plate Chambers (RPCs) around and downstream of the TPC. The purpose of the RPCs was to complement particle identification by time of flight (TOF) in particular for momenta for which the $\mathrm{d} E / \mathrm{d} x$ measurement in the TPC cannot distinguish between electrons and pions (150-250 MeV/c).

The RPCs were designed for an intrinsic time resolution of $\sim 200 \mathrm{ps}$ and intrinsic efficiency close to $100 \%$. Other constraints were an extremely short time for development and construction $^{1)}$, and low cost. The performance requirements were well matched by the multi-gap RPC concept $[1,2]$ which we adapted to our needs.

The HARP RPCs was the first large-scale RPC system employed in an experiment for precision timing. Preliminary reports on its design and performance have been presented at several conferences [3-5].

This paper also sets the record straight with respect to the RPC performance reported in Refs. [6-8]. It justifies and details our criticisms thereof $[9,10]$.

\section{RPC LAYOUT, DESIGN, AND READOUT ELECTRONICS}

The RPC system consisted of 46 identical chambers. Thirty of them, referred to in the following as barrel RPCs, were arranged in two partially overlapping layers around the TPC as shown in Fig. 1. The barrel RPCs provided for full coverage in azimuthal angle. The active volume of one RPC in the inner (outer) layer covered $14.4^{\circ}\left(14.0^{\circ}\right)$ in azimuth resulting in a $\sim 16 \mathrm{~mm}$ wide overlap of the active volumes of neighbouring RPCs. In the $z$ coordinate the active volumes of the RPCs extended from $-535 \mathrm{~mm}$ to $+1385 \mathrm{~mm}$ and covered polar angles from $17^{\circ}$ to $142^{\circ}$ with respect to the beam axis, seen from the target whose centre was located at $z=0$.

The other 16 RPCs extended the polar angle coverage in the forward direction from $16^{\circ}$ to $6^{\circ}$. They were installed $\sim 2.1 \mathrm{~m}$ downstream of the target centre, perpendicular to the beam and arranged in four groups of four RPCs each as shown in Fig. 2.

The active element of the RPC is shown in Fig. 3. It is a glass stack with four $0.3 \mathrm{~mm}$ wide gas gaps consisting of two sets of three glass plates each, placed symmetrically on both sides of the central readout electrode. The glass plates are $0.7 \mathrm{~mm}$ thick standard float glass ${ }^{2}$ with a bulk resistivity of $\sim 10^{13} \Omega \mathrm{cm}$. No special precautions for rounding off the edges were

\footnotetext{
1) The complete system was developed, constructed, and installed in nine months.

${ }^{2)}$ GLAVERBEL S.A., 116 Chaussée de la Hulpe, BE-1170 Bruxelles.
} 

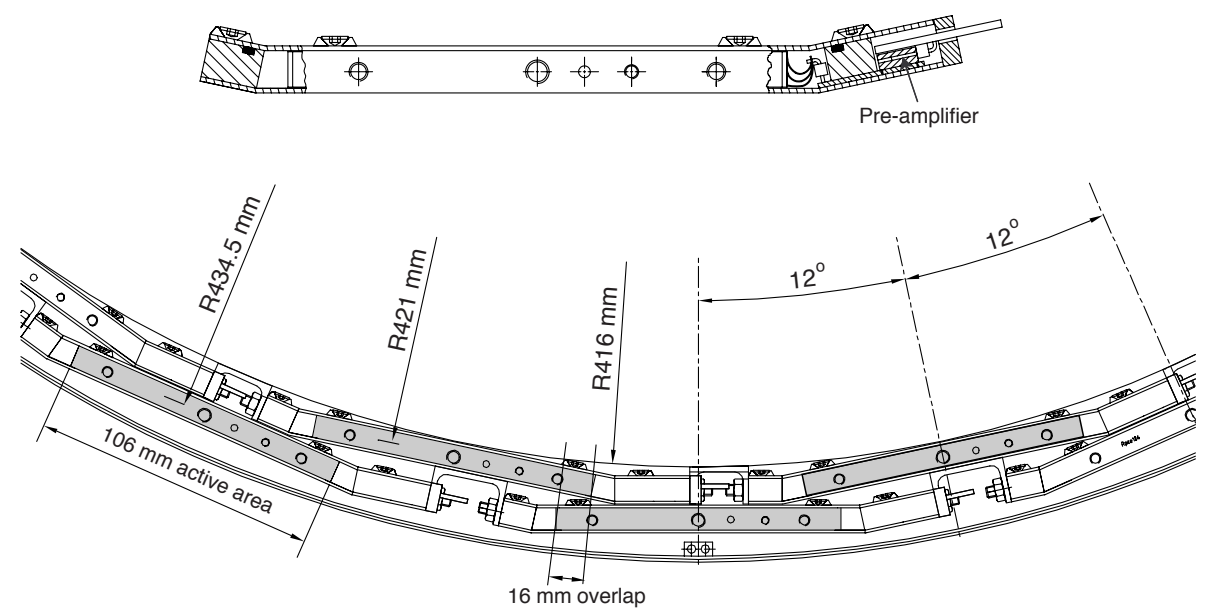

Fig. 1: Layout of a section of the barrel RPCs, looking upstream (bottom), and magnified view of one RPC (top).

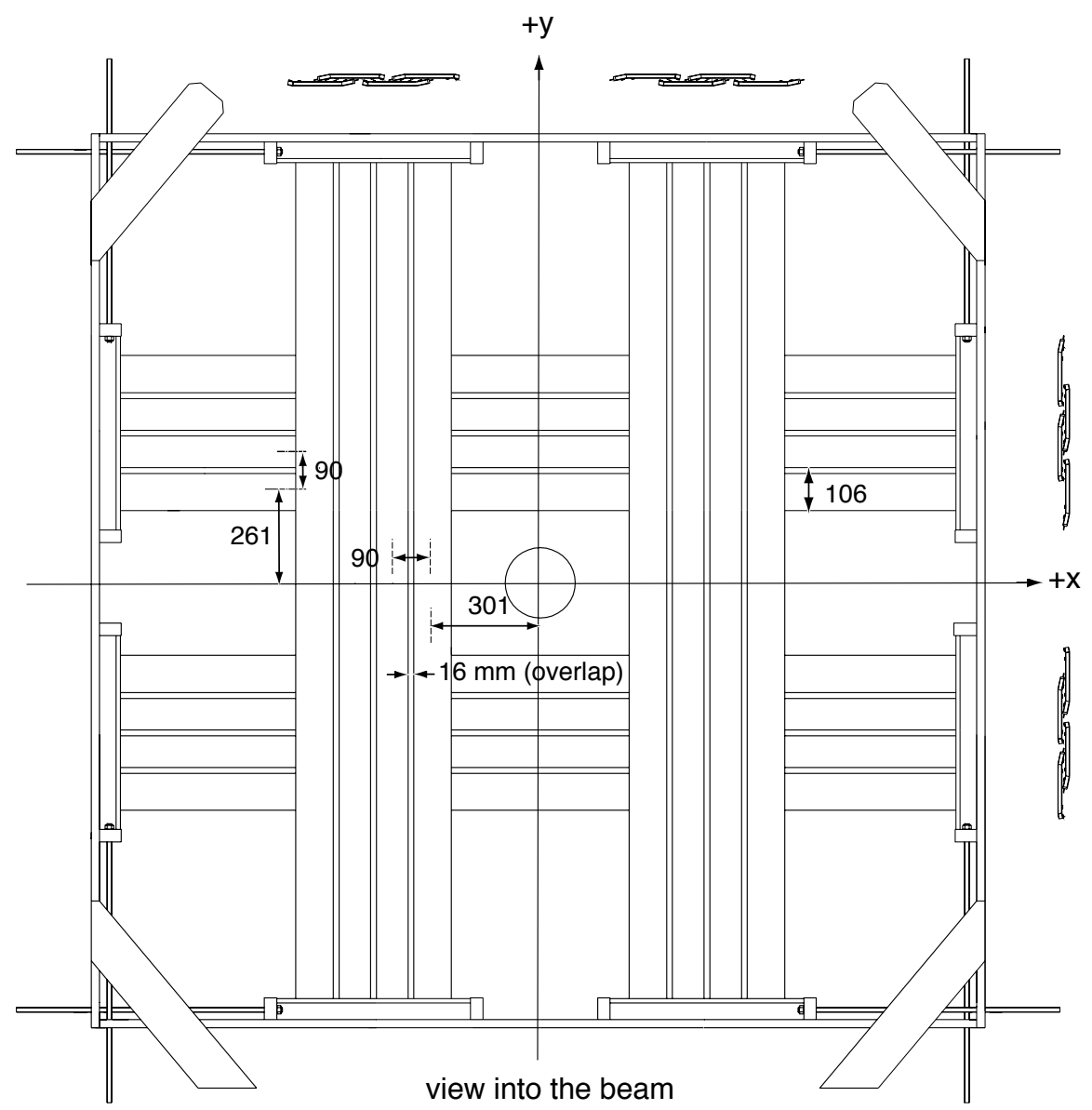

Fig. 2: Layout of the forward RPCs, looking upstream; the side and top views of the RPCs are also shown. 
necessary. The glass stack is $106 \mathrm{~mm}$ wide, $1930 \mathrm{~mm}$ long, and $7.8 \mathrm{~mm}$ thick. The narrow gaps are maintained by four $0.3 \mathrm{~mm}$ thick fishing lines per gap, stretched longitudinally, assuring a mechanical precision of the gap width of better than $10 \mu \mathrm{m}$. The six glass plates and the fishing lines are kept in position by two machined Noryl end pieces, shown in Fig. 4.

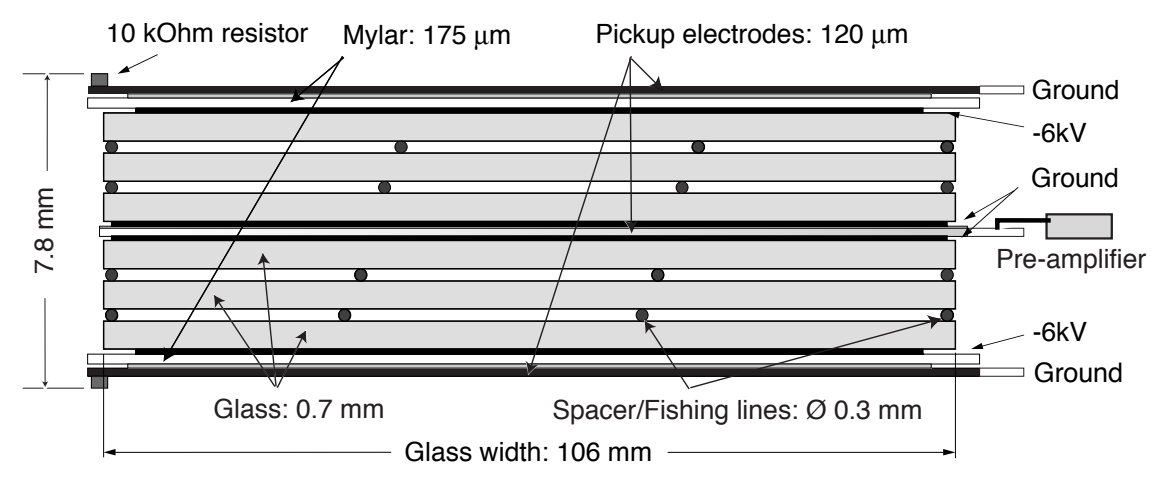

Fig. 3: Transverse cross-section of the RPC structure (different horizontal and vertical scales).

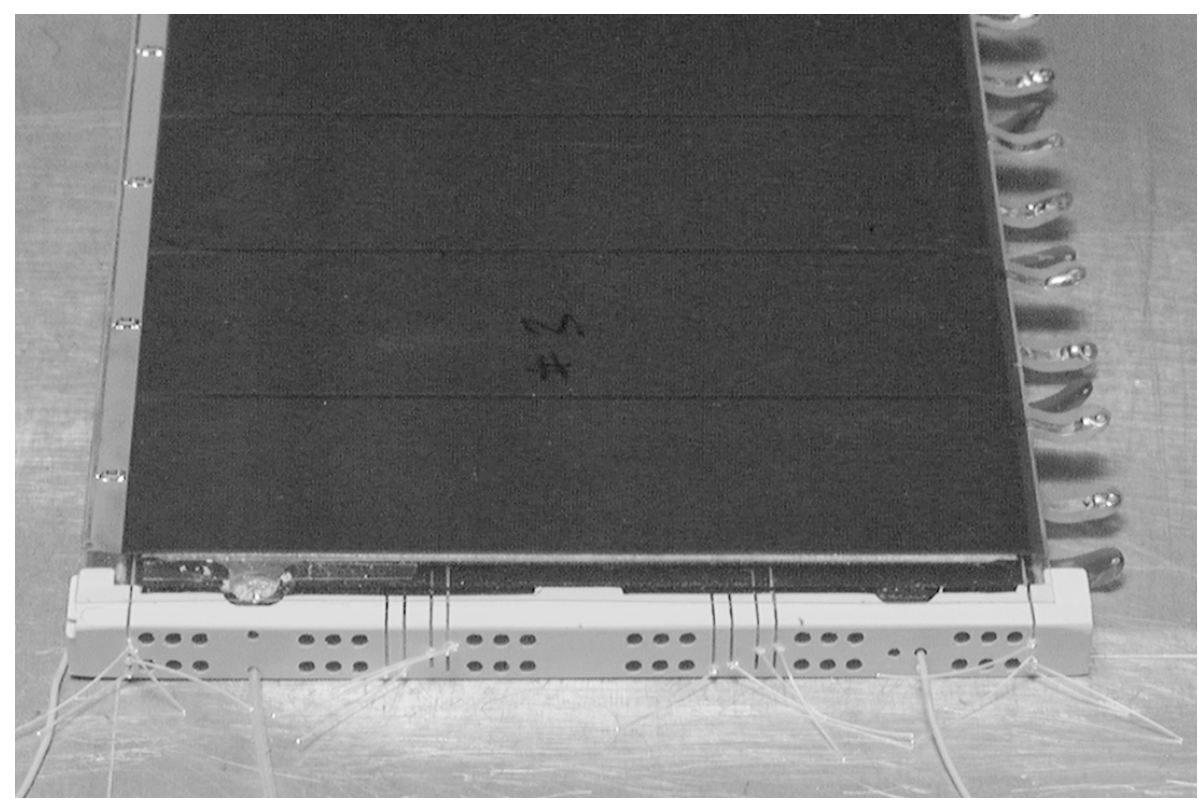

Fig. 4: Photo of the end of the RPC glass stack, showing the Noryl end pieces, the first few electrode strips, and, on the right, the strip connections to the feed-throughs (see text).

The two outer glass plates of each set were coated on the outside with graphite tape ${ }^{3)}$ with a surface resistivity of $\sim 5 \mathrm{M} \Omega /$ square. A high voltage of $-6 \mathrm{kV}$ was applied to these coatings. The two inner glass plates of each set were also coated with graphite tape, this time on the inner sides; they were connected to ground potential. The middle glass plate of each set was left electrically floating and thus adjusted itself with the continuous flow of electrons and ions to

\footnotetext{
${ }^{3)}$ ESD EMI Engineering Corporation, Toyo 5-chome, Tokyo 135-0016.
} 
its equilibrium potential. Since the resistive glass plates are electrically transparent, the analog sum of the signals of all gas gaps can be read out with one single electrode located between the two sets of plates. In addition to the central readout electrode, reference electrodes, connected to ground and electrically insulated from the HV by a $175 \mu \mathrm{m}$ thick layer of Mylar, were placed on the outside of the two glass sets.

The glass stack was housed in a gas-tight aluminium box of outer dimensions $2 \mathrm{~m} \times 150 \mathrm{~mm}$ $\times 10 \mathrm{~mm}$. The gas inlet and outlet were located at the two end faces, with the gas flowing along the length of the chamber. The high voltage was connected to the HV graphite tape at one end of the glass stack.

All electrodes ( $5 \mu \mathrm{m} \mathrm{Cu}$ on a $75 \mu \mathrm{m}$ Kapton layer) were segmented into 64 strips of dimensions $29 \mathrm{~mm} \times 104 \mathrm{~mm}$, with the strips separated by $1 \mathrm{~mm}$ from each other, as shown in Fig. 5 . The strips were connected at one end through a feed-through to the preamplifier boards. The latter were designed as plug-in modules, accessible from the outside.

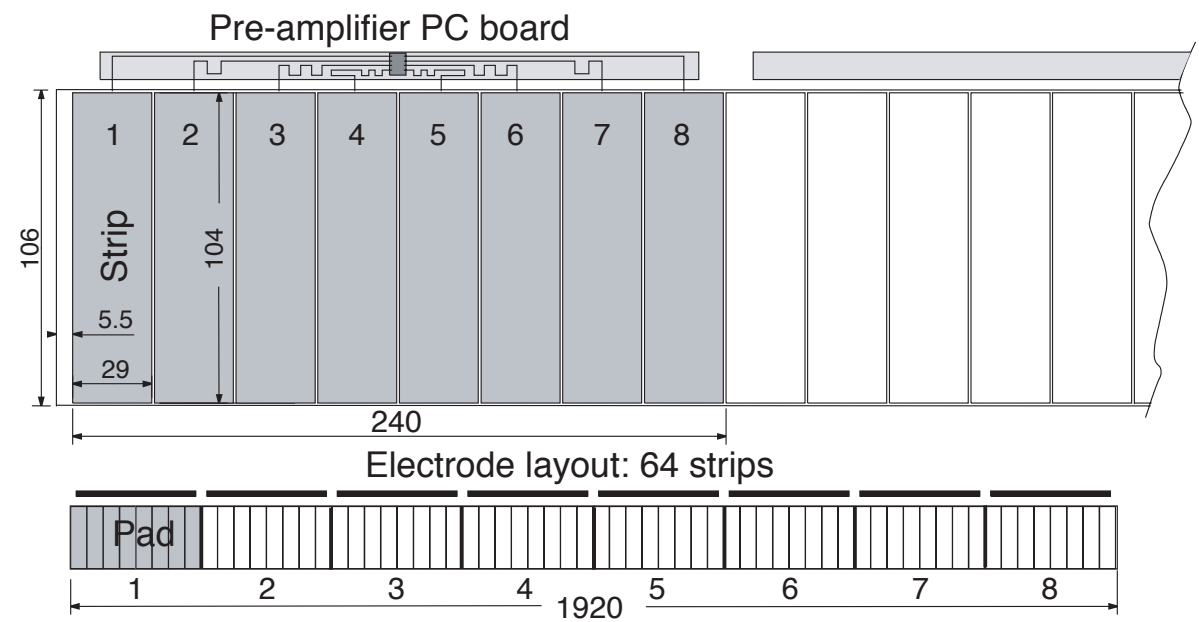

Fig. 5: Layout and dimensions of strips and pads and location of the on-chamber preamplifier printed boards. The lower figure shows all 64 strips along the full length of the RPC, eight adjacent strips form one pad.

The RPC readout electronics comprised two stages: an on-chamber preamplifier and a 'splitter' unit located at a distance of about $5 \mathrm{~m}$ from the RPCs. The preamplifier's central active element was a fast summing amplifier ${ }^{4}{ }^{4}$ which combined the signals of eight adjacent strips into one signal per "pad' ${ }^{5)}$. Thus each RPC is segmented into eight pads leading to 368 readout channels for the full system. Within one RPC the pads are numbered 1 to 8 , with pad 1 being most upstream in the barrel RPCs.

With a view to matching the strip impedance and enabling high-impedance summing of signals from adjacent strips, each strip was first connected to a low-noise fast transistor ${ }^{6}$. The transistor outputs were connected through printed-board delay lines to the input of the fast summing amplifier. The delay lines were designed to render the timing of all eight strips equal within one pad. This goal was de facto only met within $\sim 100$ ps. Figure 6 shows the relative

\footnotetext{
4) Analog Devices AD8009.

${ }^{5)}$ Each pad covers $240 \mathrm{~mm}$ in the $z$ direction.

6) PHILIPS BFR92A.
} 
delays of the time signals from all eight strips with respect to the central strips 4 and 5 of a pad, averaged over all pads. These delays are corrected for in the TOF determination.

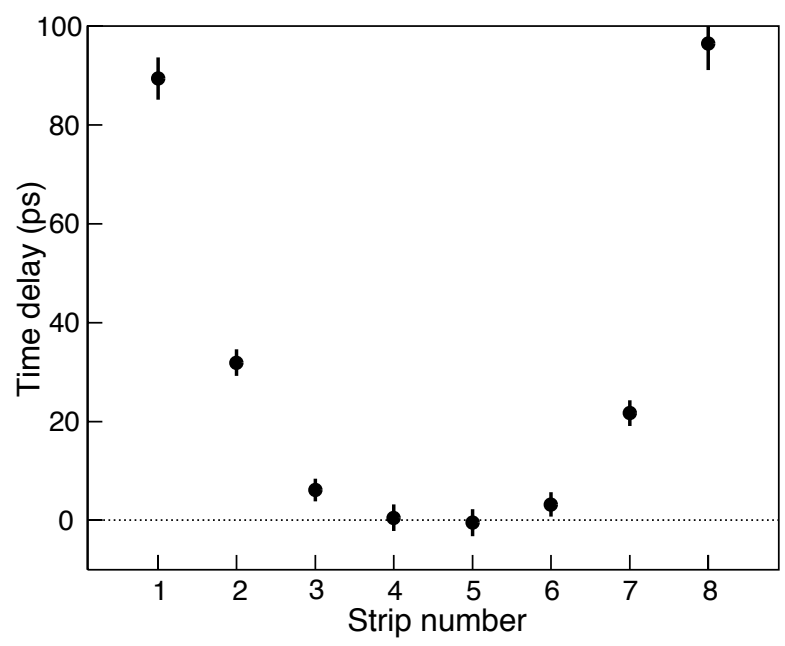

Fig. 6: Delay of the time signal of the eight strips of a pad with respect to the central strips 4 and 5, averaged over all pads.

The preamplifier signals had a rise time of $\sim 1 \mathrm{~ns}$ and a FWHM of $\sim 15 \mathrm{~ns}$. They were transmitted through mini-coaxial cables of 0.8-2.5 m length to a passive connector board and from there through $5 \mathrm{~m}$ long $50 \Omega$ coaxial cables to the splitter. Here the signals were split into two signal paths. Signals in the fast-timing path were further amplified ${ }^{7)}$ and discriminated above the noise level; the output ECL signal was sent via $80 \mathrm{~m}$ of twisted pair cable to a TDC ${ }^{8)}$. Signals in the charge path were also amplified and then sent as analog signals over $80 \mathrm{~m}$ of twisted pair cable and an impedance adapter to a QDC ${ }^{9}$.

The electronics circuitry is described in detail elsewhere [11]. In Table 1 we summarise the parameters that are relevant for the RPC timing.

Table 1: Parameters of the RPC timing electronics

\begin{tabular}{|l|c|}
\hline Amplification of the on-chamber preamplifier & $100 \mathrm{mV} / \mathrm{pC}$ \\
Equivalent input r.m.s. noise of the on-chamber preamplifier & $10 \mathrm{fC}$ \\
Equivalent threshold at the input of the on-chamber preamplifier & $50 \mathrm{fC}$ \\
Voltage amplification of the splitter & 10 \\
Overall r.m.s. noise at the splitter timing threshold & $10 \mathrm{mV}$ \\
Threshold of the splitter timing discriminator & $50 \mathrm{mV}$ \\
Pulse rise time at the splitter timing threshold & $1.8 \mathrm{~ns}$ \\
\hline
\end{tabular}

\footnotetext{
7) The transmission of the preamplifier signal through the coaxial cables and the further amplification caused a slight deterioration of the preamplifier pulse, in that the rise time increased from $\sim 1 \mathrm{~ns}$ to $\sim 1.8 \mathrm{~ns}$.

${ }^{8)}$ CAEN V775 with nominally 35 ps/count; our calibration gave slightly larger conversion constants varying from channel to channel between 34 and $38 \mathrm{ps} /$ count, with an average of $\sim 36.5 \mathrm{ps} /$ count.

${ }^{9)}$ CAEN V792 with nominally $0.1 \mathrm{pC} /$ count.
} 


\section{OPERATING CONDITIONS AND EXPERIENCE}

The RPCs were operated in avalanche mode with a gas mixture ${ }^{10)}$ of $90 \% \mathrm{C}_{2} \mathrm{~F}_{4} \mathrm{H}_{2}, 5 \% \mathrm{SF}_{6}$, and $5 \% \mathrm{C}_{4} \mathrm{H}_{10}$, and a $\mathrm{HV}$ of $-6 \mathrm{kV}$ across two gas gaps. The gas flow corresponded to approximately one volume change per hour. For practical reasons two neighbouring RPCs were connected in series.

Typical noise rates were $\leq 200 \mathrm{~Hz}$ per RPC, i.e. $\leq 1 \mathrm{kHz} / \mathrm{m}^{2}$, with a few chambers showing $2-10$ times higher noise rates ${ }^{11)}$. Over the two years of operation no signs of ageing were observed.

The operating temperature varied between $20^{\circ} \mathrm{C}$ and $35^{\circ} \mathrm{C}$. The barrel RPCs were located in a temperature-stabilized environment with temperatures of $27-30^{\circ} \mathrm{C}$, depending on their exact location but stable within $0.5^{\circ} \mathrm{C}$. The forward chambers were fully exposed to the ambient temperature in the experimental area with temperatures varying between $20^{\circ} \mathrm{C}$ and $35^{\circ} \mathrm{C}$ and day-night differences of up to $10^{\circ} \mathrm{C}$. The temperatures both for the barrel and the forward RPCs were recorded throughout the data taking by a set of 16 temperature sensors distributed across the RPCs.

As shown in Fig. 7, the RPC time response showed a strong dependence on the ambient temperature in the experimental area. Average timing drifts were $60 \mathrm{ps} /{ }^{\circ} \mathrm{C}$, with channel-to-channel
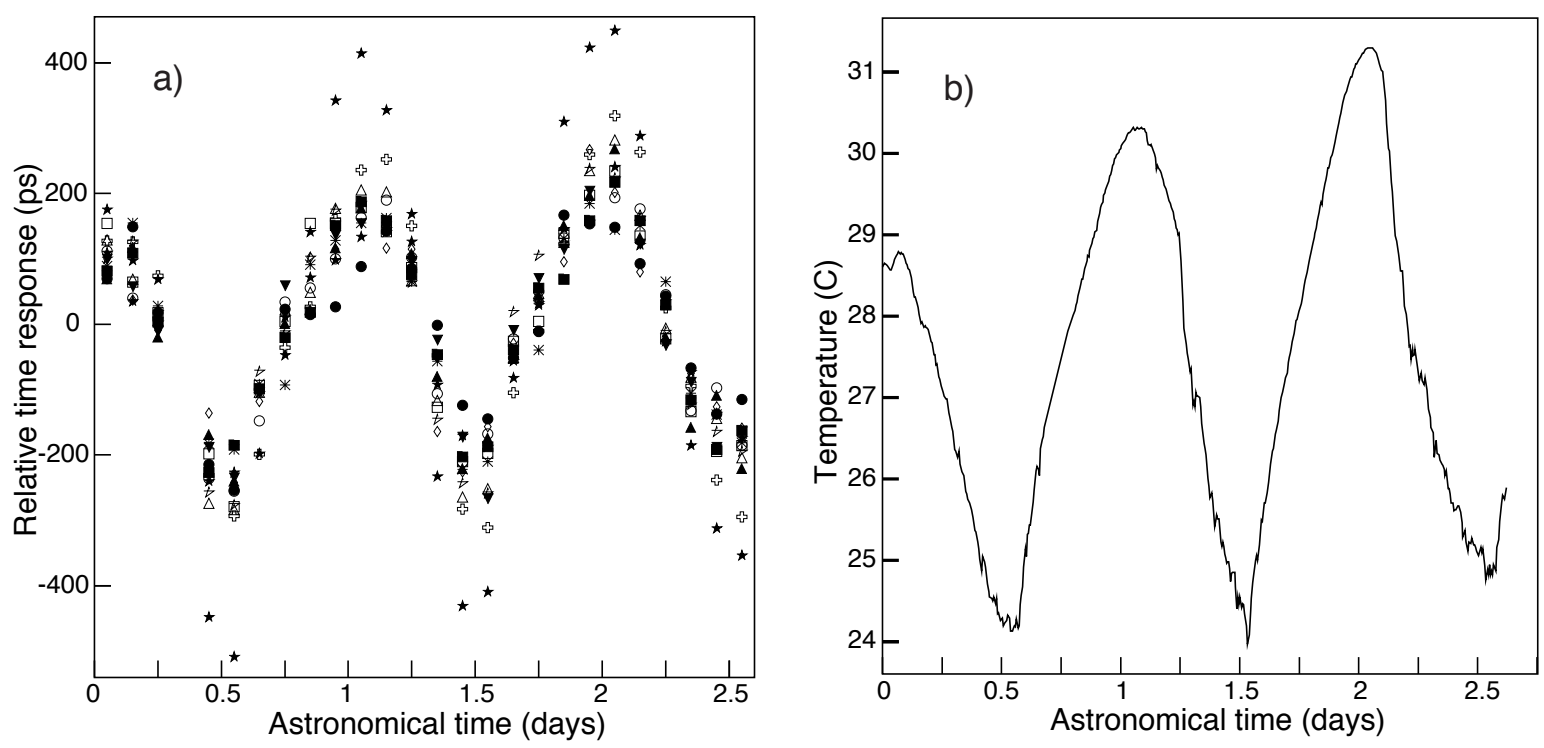

Fig. 7: Relative time response of an arbitrary selection of pads in the barrel RPCs (a) and ambient temperature in the experimental area (b) as a function of time, for the data taking period from 2002-0816 18:00 to 2002-08-19 8:00.

variations up to $20 \mathrm{ps} /{ }^{\circ} \mathrm{C}$. Since the temperatures of the barrel RPCs themselves were almost independent of the ambient hall temperature possible explanations are temperature-dependent signal transmission times in the $80 \mathrm{~m}$ long cables between discriminators and TDCs and/or

\footnotetext{
${ }^{10)}$ Because of the narrow gas gaps, the use of a heavy gas such as Freon $\left(\mathrm{C}_{2} \mathrm{~F}_{4} \mathrm{H}_{2}\right)$ is mandatory in order to ascertain a primary ionisation cluster density of $\sim 8$ clusters per millimetre.

${ }^{11)}$ Some of these chambers were opened and inspected. In all cases it turned out that the gas gaps were locally not maintained at $0.3 \mathrm{~mm}$; the fishing lines serving as spacers had locally slipped out from between the glass plates.
} 
drifts of the discriminator thresholds with temperature. The latter should lead to a charge dependence of the temperature drifts of the time signal, which we do not observe and therefore exclude as main source. Since the ambient temperature was recorded throughout the data taking, the correction of the temperature dependence posed no problem. All results given below are corrected for drifts with temperature and refer to $25^{\circ} \mathrm{C}$.

Concerning the charge response, no dependence on the temperature in the hall was seen for the barrel RPCs, consistent with their operation in a temperature-stabilized environment. For the forward RPCs we observed a small but clear increase of charge with ambient temperature of $\sim 2.2 \% /{ }^{\circ} \mathrm{C}$, see Fig. 8 .

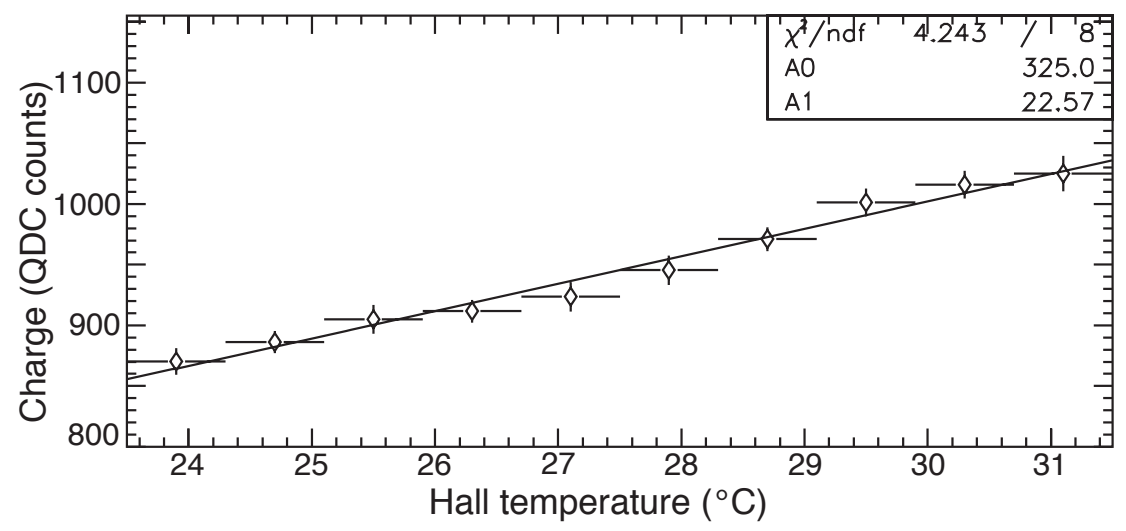

Fig. 8: Average RPC charge response for the pads in the forward RPCs as a function of temperature.

\section{INTRINSIC RPC PROPERTIES AND PERFORMANCE}

We distinguish between intrinsic RPC performance and system performance. The former refers to the performance of the RPC alone; the latter refers to its performance within its experimental environment and accounts for external factors like beam timing, particle backgrounds, nearby materials, etc.

The intrinsic chamber properties were determined in two ways: with data from a dedicated calibration scan and with physics data. The dedicated calibration scan was carried out with four spare RPCs exposed to a $-12 \mathrm{GeV} / \mathrm{c}$ momentum beam. The goal was to study the RPC response over its full active area to particles of known type $\left(\pi^{-}\right)$, momentum, and impact point. The four RPCs were placed horizontally on a moveable table, one behind the other, perpendicular to the beam. The data from the calibration scan were also used to determine global RPC characteristics.

Pad-specific properties such as the zero of the time scale $\left(t_{0}\right)$ of every RPC channel and/or deviations from the global characteristics were determined from physics data obtained in a beam of protons and pions of $+8.9 \mathrm{GeV} / c$ momentum impinging on a $5 \% \lambda_{\text {abs }}$ Be target, and of $+3 \mathrm{GeV} / c$ on a $5 \% \lambda_{\text {abs }}$ Ta target.

\subsection{Intrinsic efficiency and uniformity of response}

The intrinsic RPC efficiency and the uniformity of response were measured in the dedicated calibration scan by using two RPCs as trigger and then checking for the presence of a hit in a third, independent, RPC. Figure 9 shows efficiencies of 97-98\%, with no significant dependence 
on position within the pad. No drop in efficiency was observed at the transition from one pad to its neighbour pad when considering the OR of the pad signals.
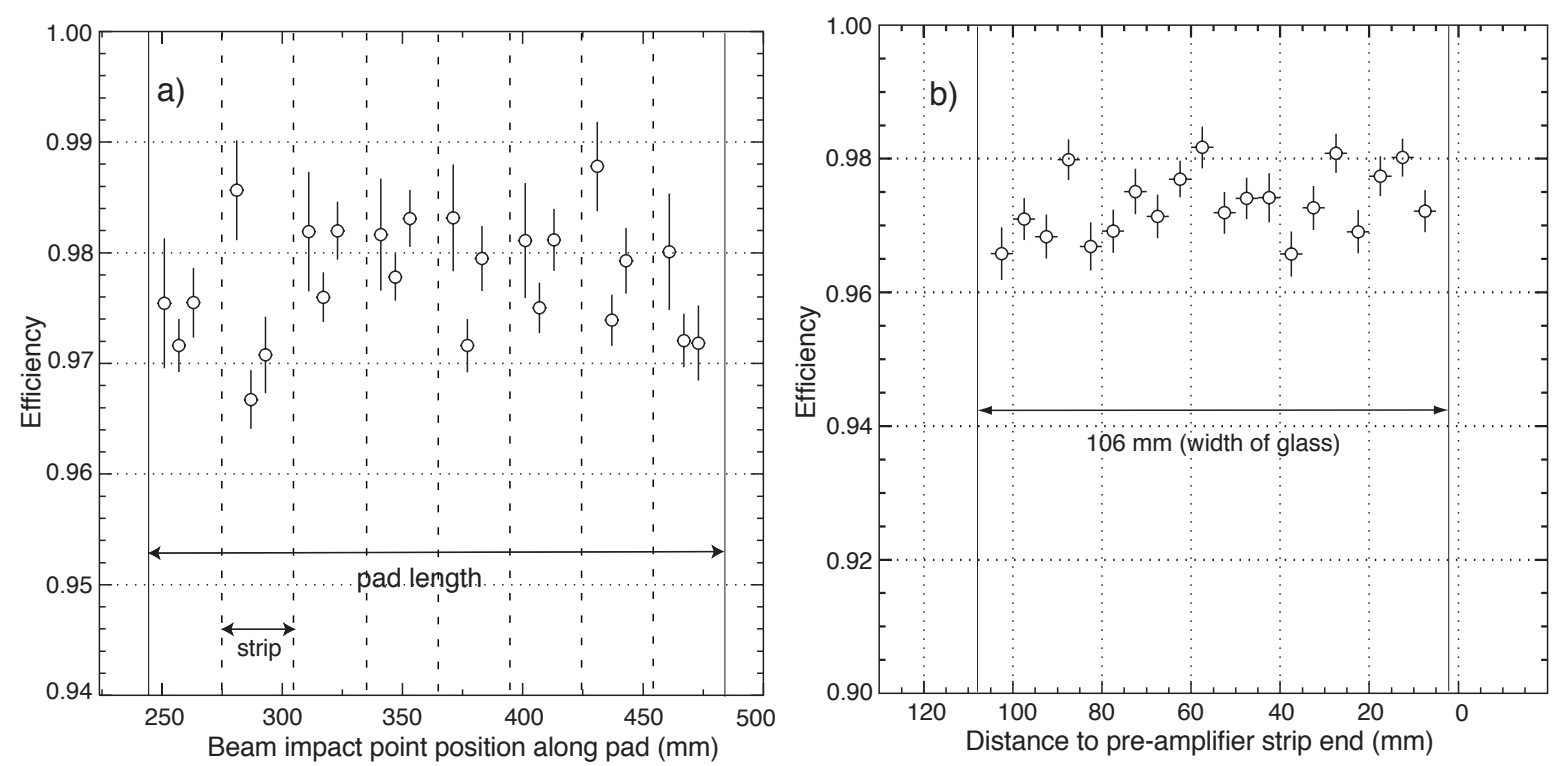

Fig. 9: RPC efficiency as measured in a dedicated calibration scan as a function of the impact position: a) along the pad across its eight strips (indicated by dashed lines); b) across a pad along a single strip.

During the dedicated calibration scan the density of incident particles was $3 \mathrm{kHz} / \mathrm{cm}^{2}$ in the centre of the beam, much higher than the rates in physics data taking $\left(\leq 1 \mathrm{~Hz} / \mathrm{cm}^{2}\right)$. An indication of a small rate dependence of the efficiency can be extracted from the left plot of Fig. 9. It shows three data points for each strip. The points in the middle correspond to the centre of the beam and thus highest particle density. Their efficiency is systematically $0.5-1 \%$ lower than for the other two points in the same strip. The intrinsic efficiency of the RPCs in a low-rate environment is therefore expected to be better than $98 \%$.

\subsection{Charge response as a function of track inclination}

The geometry of the RPC system in HARP was such that tracks from the interaction target reach the RPCs under very different angles, varying from $\sim 90^{\circ}$ for hits in pad ring 3 to $\sim 19^{\circ}$ for hits in pad ring 8 . Figure 10 shows the charge spectra produced by charged tracks in the eight RPC pad rings. The average charge is a function of the angle under which the particles traverse the RPCs, with more than twice as much charge deposited in pad ring 8 than in pad ring 3 .

\subsection{Charge attenuation}

In Fig. 11 charge spectra for tracks passing through the pad overlaps near and far from the preamplifier are given. There is no evidence for appreciable signal attenuation and signal loss at the reflection at the strip's open end ${ }^{12)}$.

\footnotetext{
${ }^{12)}$ Note that the data neither exclude nor support a small signal attenuation of $20 \%$, for example, since the resulting net amplitude difference would be $2.5 \%$ only.
} 

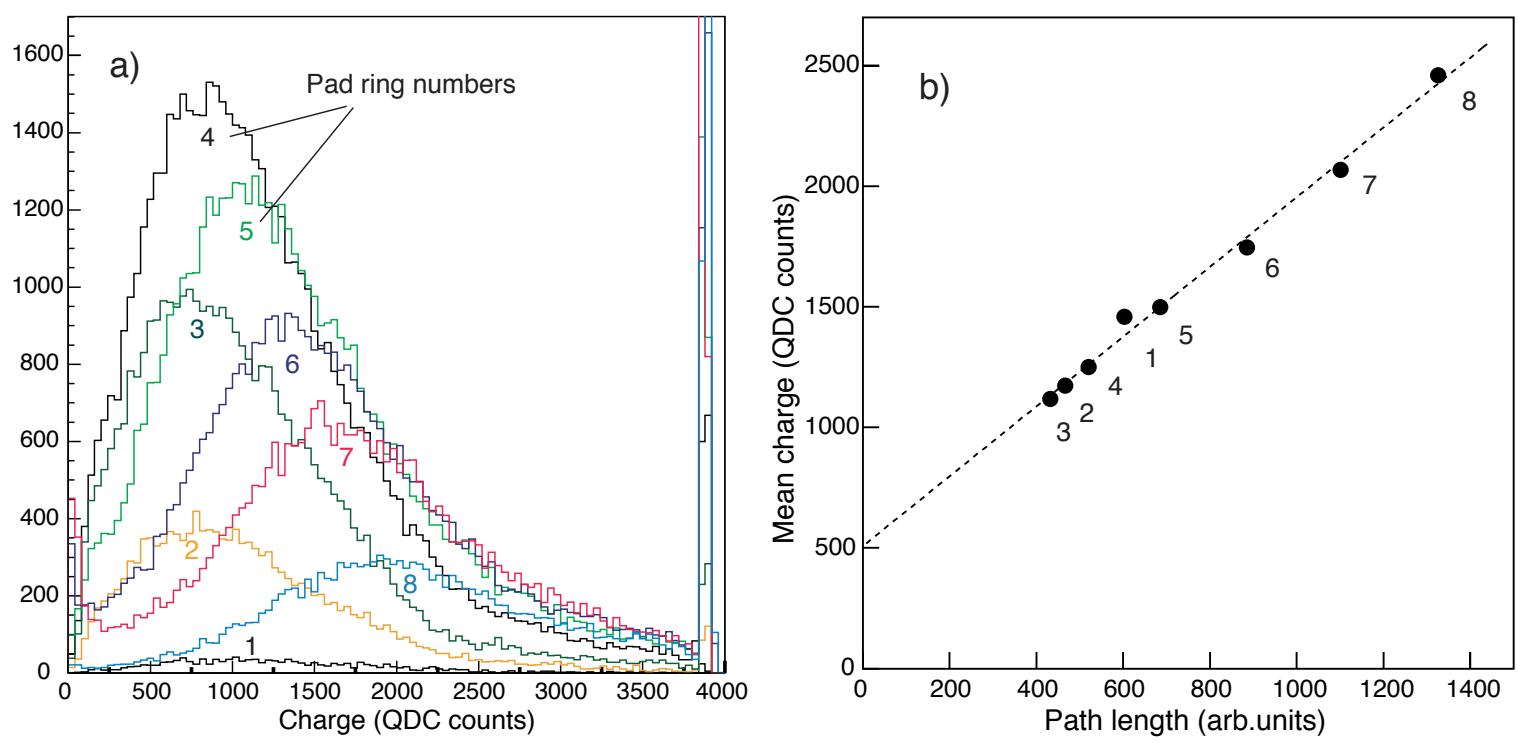

Fig. 10: Charge spectra from hits induced by charged tracks in the eight RPC pad rings (a) and average charge deposit per pad ring versus track path length through the RPC (b). The numbers in the two plots denote the pad rings, 1 is the most upstream and 8 the most downstream pad ring. The straight line is merely to guide the eye.
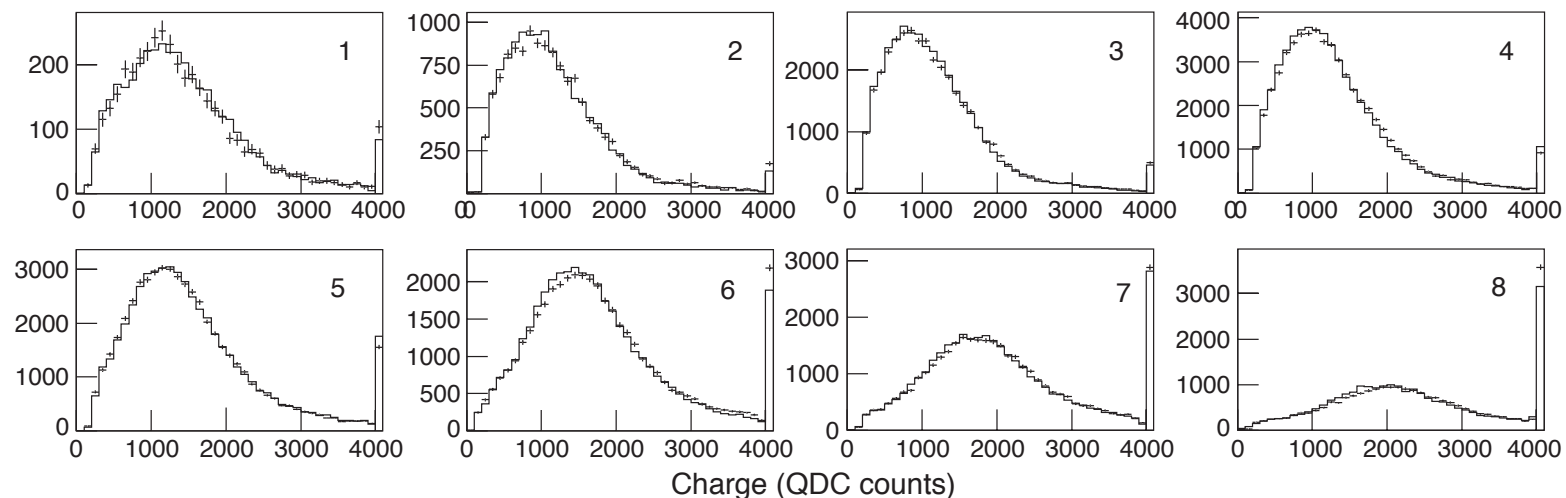

Fig. 11: Charge spectra from hits induced by charged tracks passing through the RPC overlaps near (full line) and far (crosses) from the preamplifiers, for pad rings $1-8$. 


\subsection{Global time-slewing correction}

The RPC time signal marks the time when the signal amplitude exceeds a fixed threshold in the timing path of the splitter. Because of the time slewing caused by the finite signal rise time of $1.8 \mathrm{~ns}$, a correction is needed. The characteristics of this correction is illustrated in Fig. 12. It shows the RPC time as recorded in a single RPC pad with respect to the beam timing during the calibration scan, as a function of the charge ${ }^{13)}$ of the hit. Overlayed is the functional form

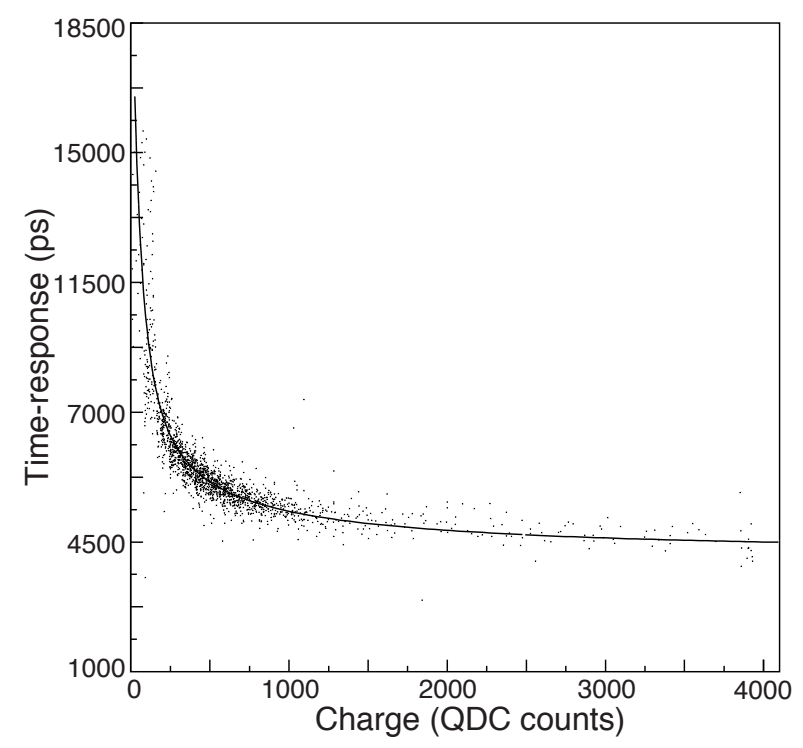

Fig. 12: Time response for a single pad as a function of charge; the charge is in QDC counts with the pedestal subtracted; overlayed is the functional form of the global time-slewing correction, as determined from the scan data.

of an average time-slewing correction, referred to below as global time-slewing correction, as determined from the scan data. It reads as

$$
\begin{aligned}
\Delta t & =a+b / Q+c / Q^{2}+d / Q^{3} \\
b & =1.5565 \times 10^{6} \\
c & =-2.8511 \times 10^{8} \\
d & =7.6798 \times 10^{10},
\end{aligned}
$$

where $\Delta t$ is the time-slewing correction in picoseconds, and $Q$ is the charge in units of QDC counts ${ }^{14)}$. The additive constant $a$ is at this stage arbitrary. Charges exceeding the maximum QDC range are only flagged as such but not measured quantitatively. Depending on the pad location, charge overflow occurs for $5-15 \%$ of the hits. For hits with charge overflow the global time-slewing correction is the one for QDC count 4095, reduced by 50-150 ps, depending on the pad ring, to account for their higher average charge.

The global time-slewing correction as determined from the calibration scan described well the time-charge behaviour of the $+8.9 \mathrm{GeV} / c$ Be data that were used for most of the systematic

\footnotetext{
13) The signal charge is for the same signal shape proportional to the amplitude.

${ }^{14)} Q$ is actually the QDC count minus the pedestal (typically 150-250 counts) plus 158.29; the latter constant was introduced to avoid a singularity at $Q=0$ when fitting the data.
} 
studies. Other data sets, taken earlier in 2002, were described better by a slightly different functional form of the global time-slewing correction.

The global time-slewing correction $\Delta t$ is subtracted from the RPC time. It is an average correction that takes out the gross effects of time slewing. It is, however, not sufficient to describe with adequate precision the time-charge relation of individual pads. This is discussed below.

\subsection{Signal response along the readout strips}

The readout strips were connected on one end only to the preamplifier. The opposite strip ends were left un-terminated in order to increase the overall signal charge. This choice led to a time response that is not only a function of the distance from the preamplifier but also of the signal charge. This is explained as follows: consider a particle impact near the preamplifier, half of the charge propagates straight to the preamplifier and triggers the time signal, the other half propagates along the strip to the opposite open strip end, is same-sign reflected and propagates back, arriving later at the preamplifier. Taking into account the pulse rise time of $1.8 \mathrm{~ns}$ at the splitter timing threshold, the timing characteristics along the readout strip depends on the signal charge. For low charge ('low' means that the time-signal delay stemming from the signal rise time is large compared with the signal transit time), the time signal tends to be independent of the position of the impact point along the strip. For high charge the time signal tends to be the more delayed the farther the impact point is from the preamplifier.

The superposition of the direct and reflected signal charges results in an apparent signal propagation time through the strip that is considerably smaller than the naive guess of 400 500 ps. Figure 13a shows the time signal as a function of the impact point along the strip, for three signal charge ranges. It has been determined with negatively charged tracks by subtracting from the times measured in the RPCs (all corrections are applied except the one for the position dependence) the times predicted from track reconstruction in the TPC. The $x$ axis gives the distance to the preamplifier: 0 corresponds to the centre of the pad overlap area close to the preamplifier ( $\sim 8 \mathrm{~mm}$ from the strip end) and 1 corresponds to the equivalent position far from the preamplifier. The time difference between far and near end is of the order of $100 \mathrm{ps,} \mathrm{with}$ an approximately linear increase from the near to the far end ${ }^{15)}$. Comparing the time signals for hits close and far from the preamplifier as a function of signal charge we observe in all pads the same characteristic behaviour, illustrated in Fig. 13b for pad ring 7. It can be parametrized as a global position- and charge-dependent correction:

$$
\Delta t_{\mathrm{pos}}[\mathrm{ps}]=t-d \cdot\left(50+Q_{\mathrm{QDC}} / 25\right),
$$

where $t$ is the time corrected for global time slewing, $d$ is the normalized distance of the hit from the preamplifier strip end, with $d=1$ for hits at the far strip end, and $Q_{\mathrm{QDC}}$ is the charge of the signal in QDC counts. The correction is applied for all charges above 300 QDC counts and below charge overflow. Correction factors for hits with charge overflow have been determined separately; they are pad-ring dependent.

\subsection{Modifications to the global time-slewing correction}

Modifications to the global time-slewing correction are intended to correct for pad-dependent deviations from the global timing corrections discussed so far. They depend on the exact signal

\footnotetext{
${ }^{15)}$ The small positive offset of the time differences stems from the fact that the data points represent the averages and not the fitted peak values of the distributions. As discussed in Sect. 5.3, the averages are slightly biased to positive values.
} 

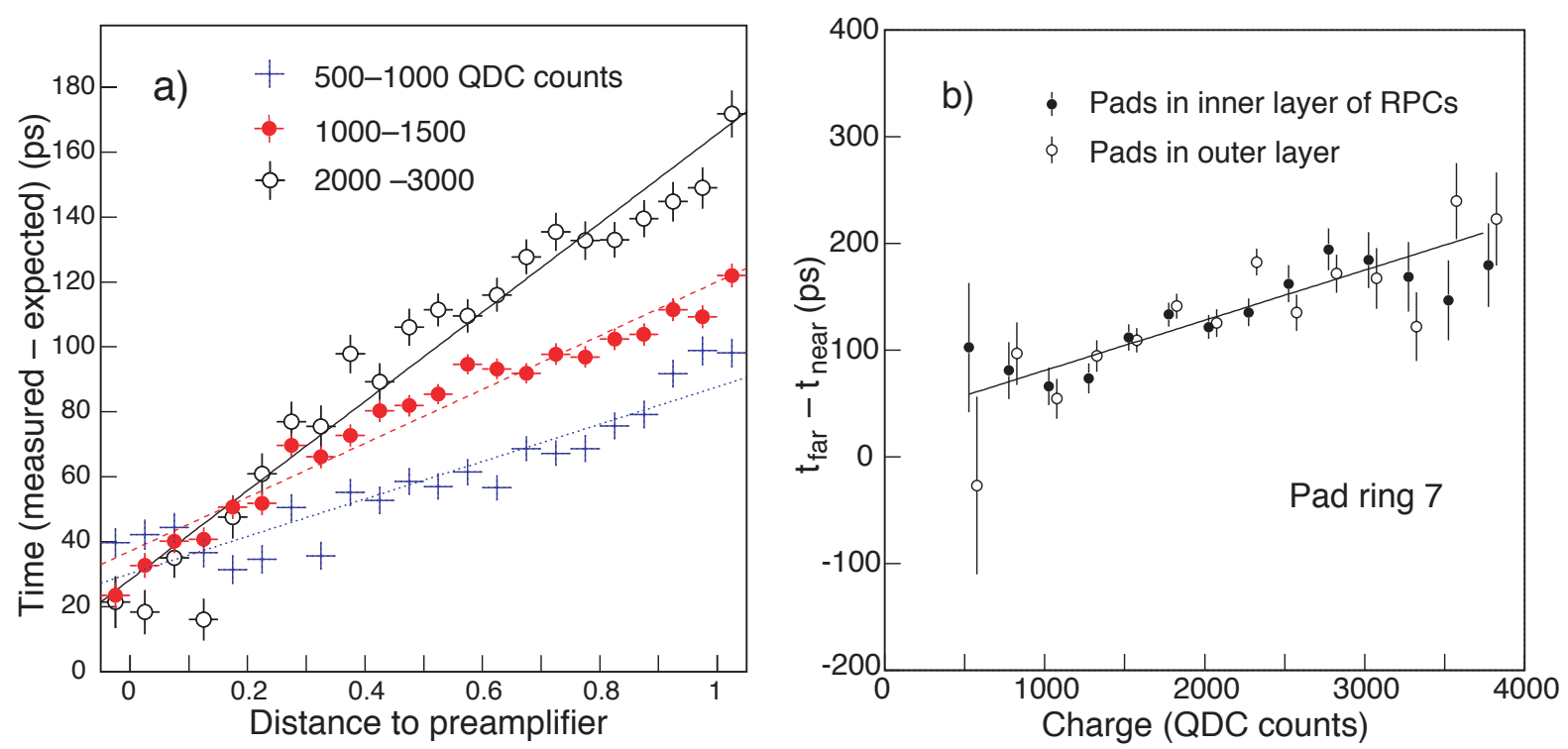

Fig. 13: a) Difference between measured and expected times for negatively charged tracks as a function of impact position along the strip for three charge bins; the lines represent linear fits to the data points. b) Difference of measured times for hits close to and far from the preamplifier as a function of the signal charge, separately for pads in the inner and outer layers of RPCs. The line shows the parametrization of the correction.

shape determined by individual electronics components and the signal transmission for different cable lengths. They also reflect discriminator threshold variations. The modifications required are usually small compared to the global time-slewing correction itself but can reach values of a few hundred picoseconds. They are obtained individually for each pad by comparing, as a function of charge, the times measured by the RPCs (after global time-slewing and all other corrections) with those expected for well-reconstructed $\pi^{-}$tracks in the TPC ${ }^{16)}$; an example is given in Fig. 14a. For charges between 300 QDC counts and 4094 (maximum QDC measurement range), the observed time differences are fitted by a linear charge dependence. For charges below 300 QDC counts (very low statistics) and for overflow charges single correction values are extracted. Figure $14 \mathrm{~b}$ shows as an example the fitted slopes of the linear charge dependence for all pads of pad ring 6 , demonstrating the need for a pad-by-pad modification of the global time-slewing correction.

\subsection{Abnormal pads and strips}

A number of pads showed a behaviour significantly different from the others. Two of the 368 pads were known to be not functioning (pads 126 and 226) and two others (45 and 110) were unstable, jumping between two distinct states of operation. In addition we found a number of strips with characteristics significantly different from the other strips within the same pad. An example is given in Fig. 15 showing the time and charge response of pad 67 as a function of the $z$ coordinate of the track impact position. It is apparent that for tracks hitting strip number 3 we measure lower charges and systematically longer times. Such a behaviour could be caused by different characteristics of the particular input transistor which connects the strip in question to

${ }^{16)} 0.25 \mathrm{GeV} / c \leq p_{\mathrm{T}} \leq 1.2 \mathrm{GeV} / c$. 

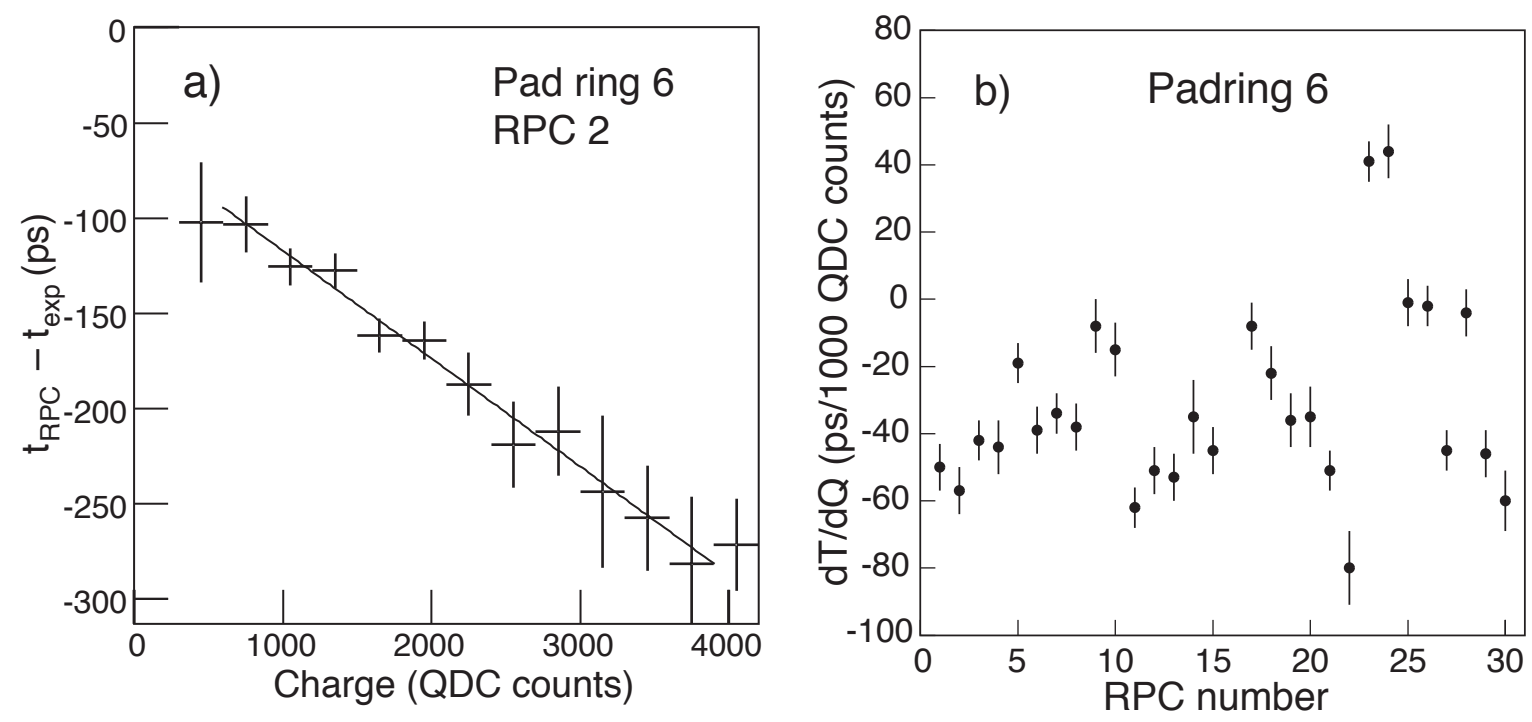

Fig. 14: Corrections to be applied on top of the global time-slewing correction as a function of charge for a single pad in pad ring 6 (a); slopes of the correction function for all 30 pads in pad ring 6 (b).

the preamplifier, or a bad contact leading to smaller charge signals for hits in this particular strip. Since the time-slewing correction is determined for the full pad, it will not correctly describe the timing for this strip.

Given that the time resolution for very low signal charges, where the time-charge relation is steeply falling, is considerably worse than for normal charges, we do not attempt to correct for these cases. In the barrel, 48 out of the 1920 strips are affected; these strips are flagged and not used further.

The four not functioning pads and the abnormal strips together correspond to $4 \%$ of the barrel area for which no RPC particle identification is available.

\subsection{Intrinsic time resolution}

The intrinsic time resolution of the RPCs was determined with tracks through the pad overlaps. For these tracks the time of flight is measured twice in two neighbouring pads. From the spread of the time differences we obtain the convoluted time resolution of the two pads involved. Any uncertainties related to the beam timing cancel. Potential errors in the 'zero times' of the individual pads $\left(t_{0}\right)$ would lead to a systematic shift of the time difference but not to a broadening of the distribution. In order to become independent of the $t_{0}$ 's, the distributions of all overlaps have been artificially shifted to be centred at zero. The resulting distribution of the time differences is given in Fig. 16a. It shows the spread of the time differences for all tracks through pad overlaps, excluding only abnormal pads and strips. The distribution is well fitted by a narrow Gaussian on top of a wider Gaussian that represents a small background of larger time differences. The wider Gaussian is consistent with the effect of back-scattering (as discussed below). We quote as intrinsic time resolution of the RPCs the width of the narrow Gaussian divided by $\sqrt{2}$. It is on average 127 ps and given in Fig. 16b as a function of the pad ring number, for pion and proton tracks separately. The time resolution improves with increased path length of the tracks through the pads; the best average time resolution of $120 \mathrm{ps}$ is obtained for pad ring 8 . We also observe a slightly better resolution for proton tracks. 


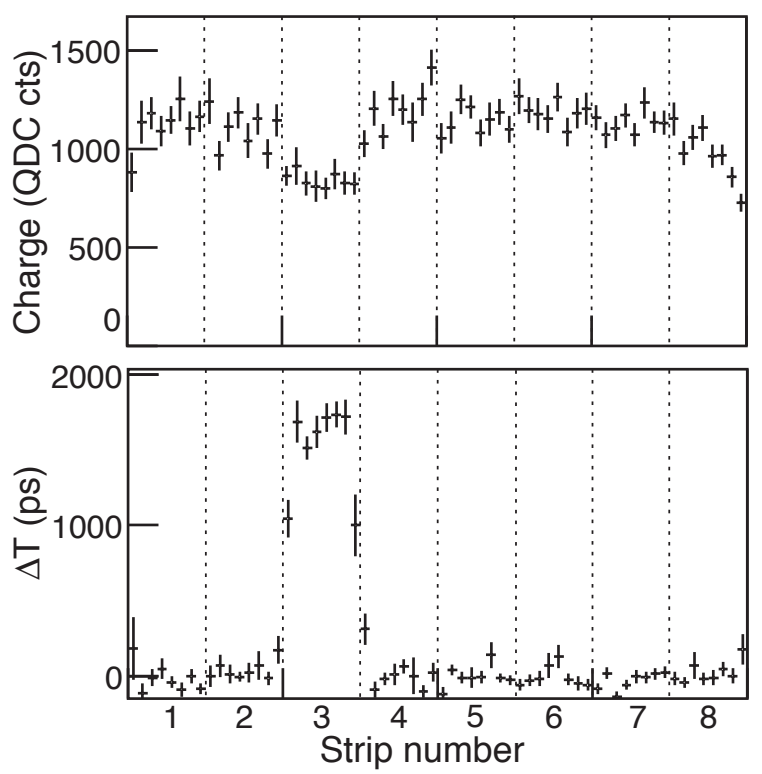

Fig. 15: Difference between measured and expected time of flight for negative pions (bottom) and the average charge for these hits as measured in the RPCs (top) as a function of the $z$ coordinate of their impact point in a single pad; the dotted lines indicate extension of the eight readout strips.
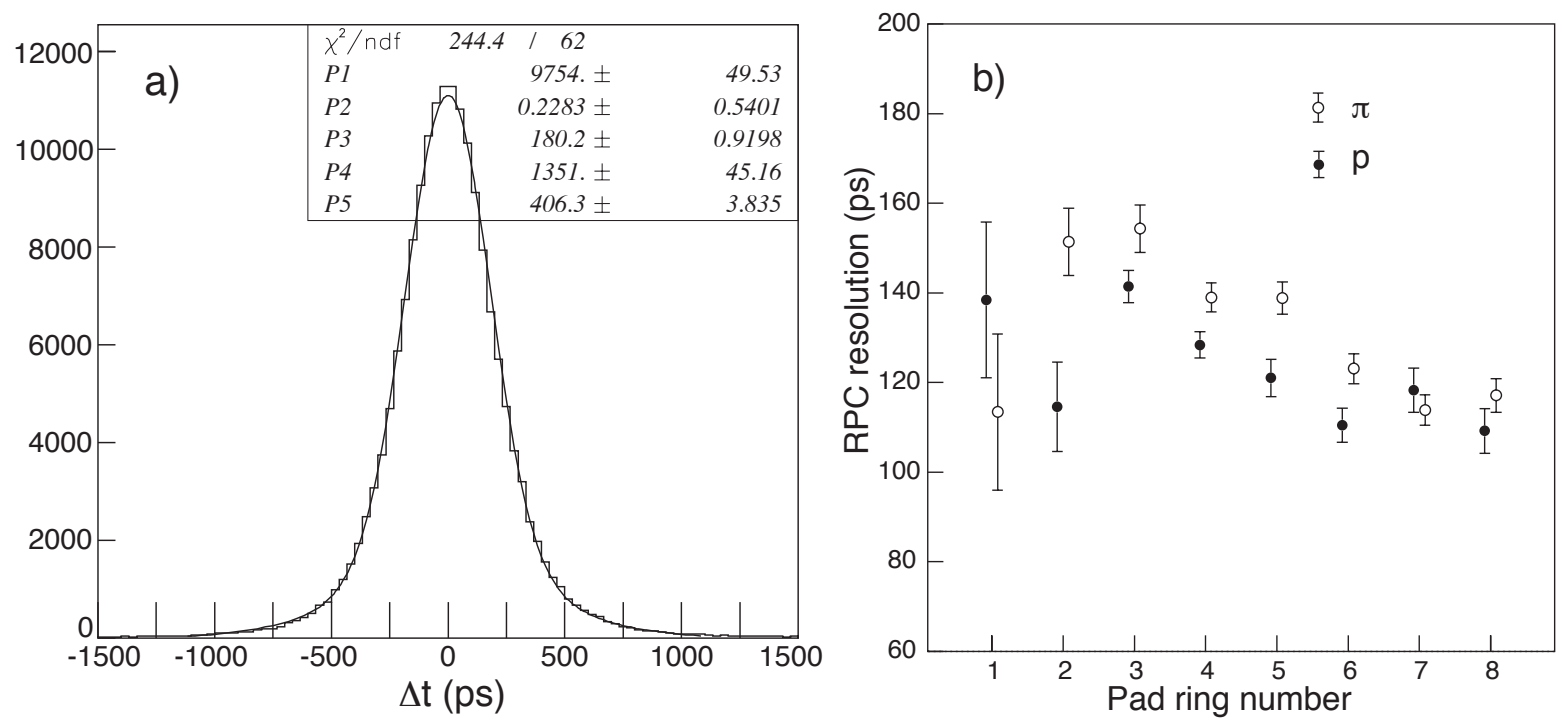

Fig. 16: a) Distribution of the differences between the two times measured for tracks passing through the pad overlap regions of the barrel RPCs; the experimental distribution is fitted by the sum of a narrow Gaussian and a wider Gaussian whose normalisations (P1, P4) and standard deviations (P3, P5) are stated; the narrow Gaussian's standard deviation divided by $\sqrt{2}$ is $127 \mathrm{ps}$; b) average intrinsic time resolution as measured with pion and proton tracks in the eight pad rings of the barrel RPCs. 
It is instructive to study the time resolution in each pad ring as a function of the signal charge. For this purpose we first selected tracks where both contributing pads had signal charges of 1000-2500 QDC counts and determined the time resolution for the corresponding pad ring as the width of the narrow Gaussian divided by $\sqrt{2}$. We then selected tracks where one of the two overlapping pads had a signal charge in the range 1000-2500 QDC counts and plotted the de-convoluted time resolution against the charge measured in the other pad. Figure 17 shows the resulting time resolution as a function of signal charge in the eight pad rings. The data show the same systematic pattern for all eight pad rings:

- The time resolution improves with increasing charge from values of $\sim 250$ ps for very low charges (with almost no data) to reach $\sim 100$ ps for charges corresponding to the maxima of the charge distributions (see Figs 10 and 11).

- The time resolution worsens again for large charges in the tails of the charge distributions.

The latter affects only a small fraction of the tracks. For those tracks, the measured charge, most likely, does not correspond to the signal charge induced by the hit for which the time was measured. Additional charge induced by back-scattered particles can explain the observed behaviour; this is discussed in more detail in Section 5.1.

We conclude that the intrinsic time resolution of the four-gap RPCs improves with the signal charge and reaches, in the absence of back-scattering, values as low as $\sim 100$ ps for charges above $\sim 1500$ QDC counts.

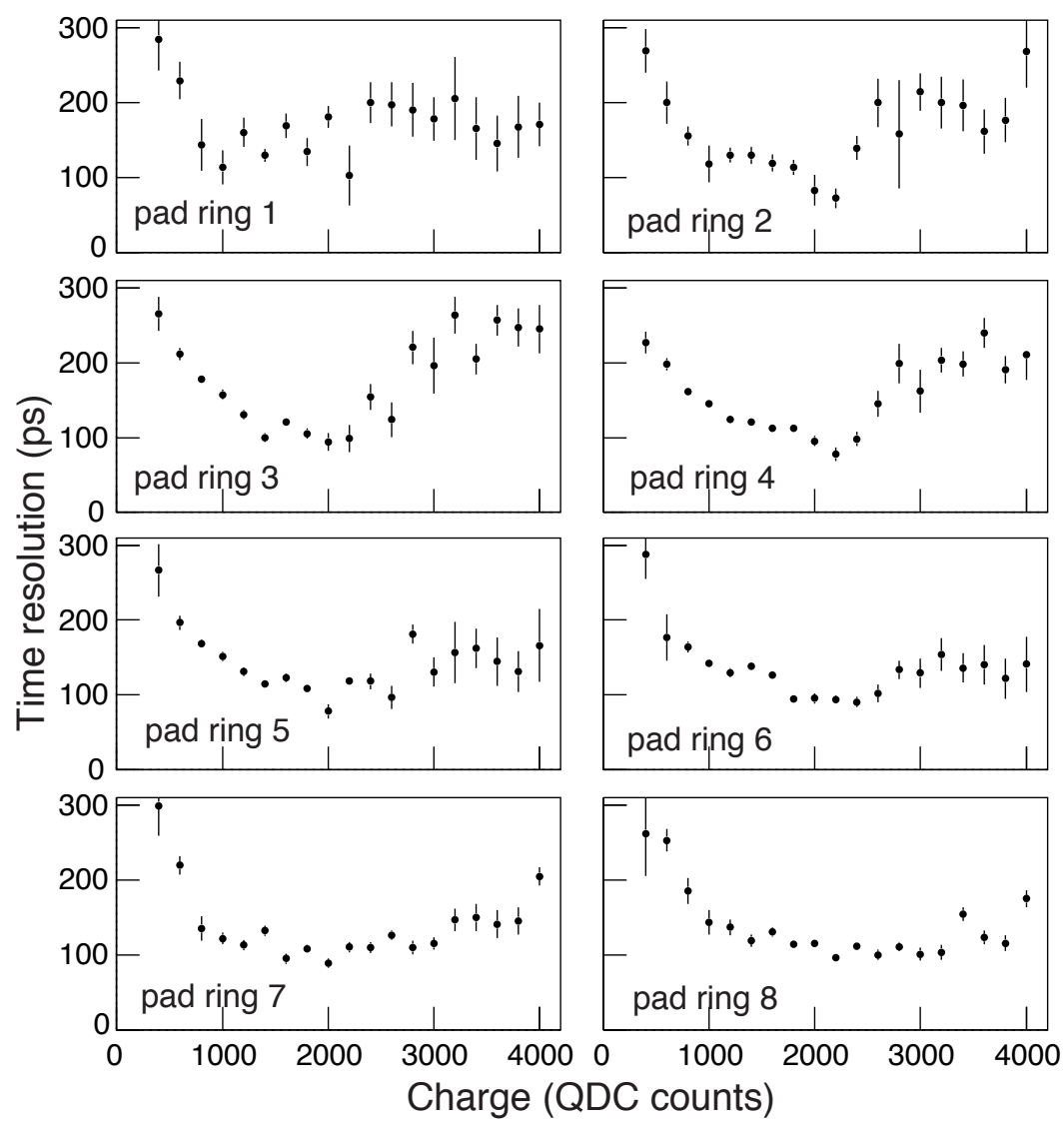

Fig. 17: Average time resolution as a function of signal charge in the eight pad rings. 


\subsection{Theoretically expected timing performance}

In this section we compare the observed timing performance with that expected from the physics of signal formation in this RPC type.

The static electric field strength in the gas gaps is $100 \mathrm{kV} / \mathrm{cm}$. In this strong field, electron multiplication leads after initial random fluctuations to an exponential increase of the number of electrons, $\propto \exp \left(\alpha_{\text {eff }} l\right)$, with $\alpha_{\text {eff }}=\alpha-\eta$, where $\alpha$ is the Townsend coefficient, $\eta$ the attachment coefficient, and $l$ the distance from the start of the avalanche. For our gas and electric field the 'effective' Townsend coefficient is $\alpha_{\text {eff }} \sim 1100 \mathrm{~cm}^{-1}$, the attachment coefficient $\eta \sim 60 \mathrm{~cm}^{-1}[12,13]$.

For an avalanche initiated by a single electron the current induced by the fast-moving avalanche electrons, ignoring the slowly moving ions, is given by [13]

$$
i(t)=I \exp \left(\alpha_{\mathrm{eff}} v t\right)
$$

where $v$ is the average electron drift velocity, and $I$ an initial current amplitude the probability of which is exponentially falling [13,14]: $\propto \exp \left(-I / I_{\mathrm{av}}\right)$. The current does not depend on the electron's position in the gas gap. The average drift velocity has for our gas and electric field the numerical value $v \sim 200 \mu \mathrm{m} / \mathrm{ns}[12,13]$.

The trigger time, that is the time when the trigger threshold ${ }^{17)} I_{\text {trig }}$ is crossed by the exponentially increasing current, is given by

$$
t_{\text {trig }}=\frac{1}{\alpha_{\text {eff }} v} \ln \left(I_{\text {trig }} / I\right)
$$

In our gas, we expect $\sim 8$ primary electron clusters per millimetre for a minimum-ionizing particle, with an average 'cluster size' of 2.3 electrons per cluster $[12,13]$. This leads for our four-gap RPC to the expectation of $\sim 13$ for the number of primary electrons, $N_{\mathrm{el}}$. This holds for a track crossing perpendicularly the RPC, and takes into account a reduction of the effective gap width by the avalanche length at the time of threshold crossing.

Each primary electron develops its own avalanche independently of the others. In the approximation that all primary electrons are produced at the same time, the trigger time $t_{\text {trig }}$ becomes

$$
t_{\text {trig }}=\frac{1}{\alpha_{\text {eff }} v} \ln \left(I_{\text {trig }} / \sum_{k=1}^{N_{\mathrm{el}}} I_{\mathrm{k}}\right) .
$$

We need to convert the trigger threshold in terms of current, $I_{\text {trig }}$, to the trigger threshold in terms of charge, $Q_{\text {trig. }}$. The time-dependent current $i(t)$ in terms of the number of avalanche electrons $N(t)$ at the time $t$ reads as $[13,15,16]$

$$
i(t)=\frac{E}{V} v e N(t)
$$

Here $E$ is the dynamic electric field in the gas gaps when a high voltage $V=6 \mathrm{kV}$ is applied across two gas gaps, and $e$ the unit electric charge. With the permittivities $\epsilon_{\text {glass }}=8$ and $\epsilon_{\text {gas }}=1$ (both in units of the permittivity of free space), and the thicknesses $d_{\text {glass }}=0.7 \mathrm{~mm}$

\footnotetext{
${ }^{17)}$ The trigger threshold for the time signal is supposed to be set in the region of exponential growth, well above the initial random fluctuations, and below the region where charge saturation in the electron multiplication process becomes dominant.
} 
and $d_{\text {gas }}=0.3 \mathrm{~mm}$, it follows from the continuity constraint for the normal component of the electrical displacement vector that

$$
\frac{E}{V}=\frac{\epsilon_{\text {glass }}}{3 \epsilon_{\text {gas }} d_{\text {glass }}+2 \epsilon_{\text {glass }} d_{\text {gas }}}=11.6 \mathrm{~cm}^{-1} .
$$

Equating the integrated current with the trigger threshold in terms of charge, $Q_{\text {trig, }}$ gives the trigger time

$$
t_{\text {trig }}=\frac{1}{\alpha_{\text {eff }} v} \ln \left(\alpha_{\text {eff }} \frac{V}{E} \frac{Q_{\text {trig }}}{e N_{\mathrm{el}}}\right) .
$$

We summarize in Table 2 the microscopic parameters of signal formation in our four-gap RPC.

Table 2: Microscopic parameters of the RPC signal formation

\begin{tabular}{|l|c|}
\hline Mean time between electron collisions $1 /\left(\alpha_{\mathrm{eff}} v\right)$ & $45 \mathrm{ps}$ \\
Mean distance between electron collisions $1 / \alpha_{\mathrm{eff}}$ & $9 \mu \mathrm{m}$ \\
Mean electron multiplication factor for m.i.p. threshold crossing & $2 \times 10^{6}$ \\
Mean time needed for m.i.p. threshold crossing $t_{\text {trig }}^{\text {m.i.p. }}$ & $660 \mathrm{ps}$ \\
Avalanche length until m.i.p. threshold crossing & $130 \mu \mathrm{m}$ \\
\hline
\end{tabular}

The number of primary electrons in the ionization by protons will be larger by the ratio of the proton to pion energy losses at the same momentum. From Eq. (3) follows that the trigger time for a proton will be shorter by

$$
\Delta t=\frac{1}{\alpha_{\text {eff }} v} \ln \frac{\mathrm{d} E / \mathrm{d} x_{\text {proton }}}{\mathrm{d} E / \mathrm{d} x_{\text {pion }}},
$$

independently of the trigger threshold. This difference is shown as full line in Fig. 18 as a function of particle momentum. The proton time signal arrives typically a few tens of picoseconds earlier than the pion time signal. The time difference increases with smaller particle momentum and amounts to $\sim 120 \mathrm{ps}$ for a momentum of $200 \mathrm{MeV} / \mathrm{c}$.

Up to now we assumed that the induced signals from all avalanches arrive at the same time. While this is a good approximation for the avalanches within one gap (we merely neglect the propagation time of the electromagnetic field across the few millimetres between the pick-up electrodes), the average time delays of signals from subsequent gaps with respect to the first gap start to matter for particles that cross the RPC with a velocity considerably lower than the velocity of light. The time difference between gaps reflects itself in a signal amplitude difference. Figure 18 shows also the result of a Monte Carlo simulation of the proton-pion timing difference which takes besides statistical fluctuations the time differences between the gaps into account.

The trigger time given by Eq. (2) fluctuates because of the fluctuation of the initial amplitude $I$. Folding in the latter's exponentially falling distribution around the average amplitude $I_{\text {av }}$, the time resolution for the single-electron case follows as [13]

$$
\sigma_{\mathrm{t}}=\frac{1.28}{\alpha_{\mathrm{eff}} v}=58 \mathrm{ps}
$$




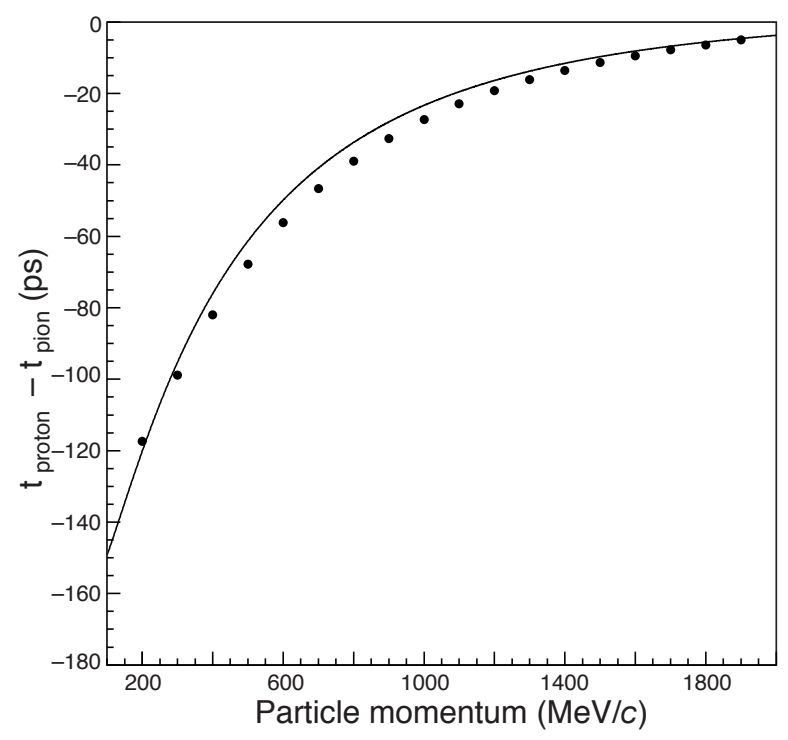

Fig. 18: Time difference [ps] by which the proton trigger time is earlier than the pion trigger time, as a function of particle momentum $[\mathrm{MeV} / \mathrm{c}]$ in the RPC; the full line shows the approximation of Eq. (4), the points the results of a Monte Carlo simulation.

The time resolution does not depend on the trigger threshold if set in the region of exponential avalanche growth.

For several primary electrons, the time resolution will improve with respect to Eq. (5), but not quite as much as naïvely expected. The reason is that a few large pulse heights will determine the time of threshold crossing, while even many small pulse heights will have little effect. In a Monte Carlo simulation, we used a Poisson-distributed number of clusters together with a $1 / n^{2}$ distribution of the cluster size, and obtained an expected time resolution of $\sim 45 \mathrm{ps}$ and $\sim 40$ ps for $1 \mathrm{GeV} / c$ pions and protons, respectively [17].

The above considerations are not affected by the transfer function of linear readout electronics. Hence the timing characteristics determined by the crossing of the equivalent threshold (50 fC, see Table 1) are fully preserved. Saturation effects in the electron avalanche formation have no influence on the timing as long as the equivalent threshold is set in the region of exponential avalanche growth.

These 'intrinsic' RPC timing characteristics are, however, buried underneath the much larger 'electronics' time slewing that is caused by the $1.8 \mathrm{~ns}$ rise time of the electronics pulse at the trigger threshold in the splitter.

In principle, the electronics time-slewing correction is completely independent of the intrinsic RPC timing characteristics discussed above. In practice, this is only approximately the case since the time-slewing correction is obtained with particles passing the RPC, with the arrival time of the beam particle at the target as external time reference. Therefore the time-slewing correction constitutes effectively a convolution of the large electronics time slewing with the small contribution from the 'intrinsic' RPC timing characteristics.

The effect of the intrinsic time difference between protons and pions, shown in Fig. 18, on the actual times measured for protons is illustrated in Fig. 19. It gives the prediction of $\beta$ by a Monte Carlo simulation for protons with an average polar angle of $\theta=50^{\circ}$ on top of the theoretical curves for particle velocity $\beta$ versus momentum. Hardly any difference is visible. 
We emphasize that the momentum variable in Fig. 19 refers to the particle momentum in the RPC, as opposed to the particle momentum in the TPC (which is larger because of the energy loss in structural material between the TPC and the barrel RPCs).

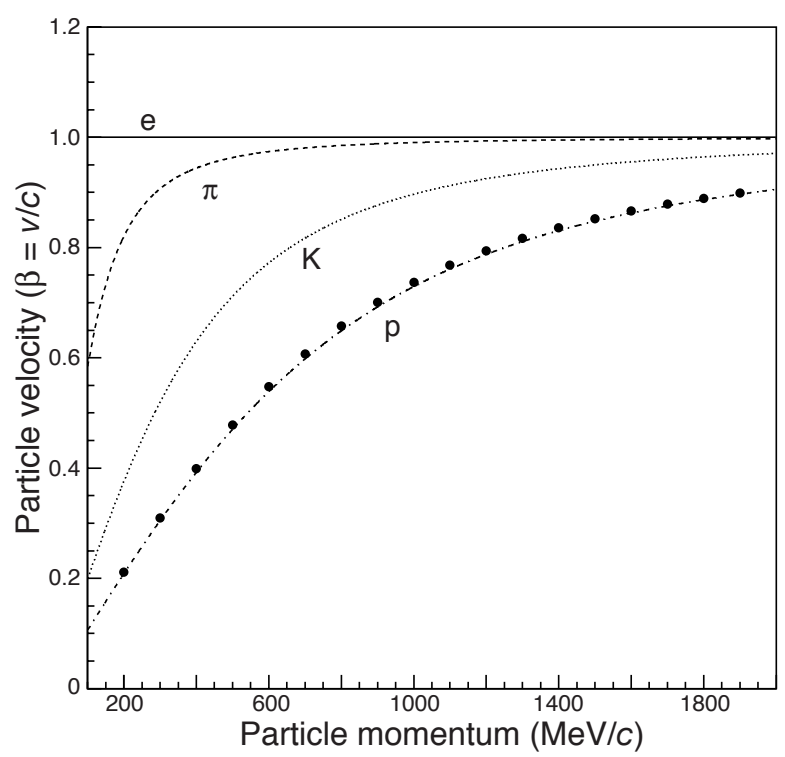

Fig. 19: Particle velocity $\beta$ versus momentum in the RPC; the points show the $\beta$ of protons as predicted by a Monte Carlo simulation.

Our observed intrinsic time resolution for pions is on average $127 \mathrm{ps}$. Even the best values of 100 ps, see Fig. 17, are considerably worse than the theoretically expected time resolution. We first note that the equivalent electronics noise at the equivalent threshold has a negligible effect on the time resolution. However, it has a non-negligible effect through the $1.8 \mathrm{~ns}$ risetime of the electronics pulse at the splitter timing threshold where it leads to a timing jitter of order 100 ps. Therefore we conjecture that the largest contribution to our observed average time resolution arises from amplifier noise ${ }^{18}$. This conjecture leads to the prediction that our RPC time resolution should improve with the inverse amplitude (or charge) of the electronics output pulse - which is indeed observed in Fig. 17, though only for small charges ${ }^{19)}$.

\section{SYSTEM ASPECTS AND PERFORMANCE}

Under system aspects we understand effects which influence the performance of the RPCs in their specific environment in the HARP detector. They comprise geometrical effects, implementation-specific backgrounds, as well as detector calibration ( $t_{0}$ determination). The final performance of the RPC system must take these into account in addition to external parameters like the beam timing accuracy and the track reconstruction quality in the TPC.

\subsection{Particle back-scattering}

The barrel RPCs are located in the narrow gap between the outer TPC field cage and the coil of the solenoidal magnet, with the $\mathrm{Cu}$ coil at a radial distance of only 5-15 $\mathrm{mm}$ from the RPCs.

\footnotetext{
18) This is consistent with the fact that we observe for protons only a slightly better time resolution than for pions.

${ }^{19)}$ The observed worsening of the time resolution for large charges has a completely different origin, namely particle back scattering, and is discussed in Section 5.1.
} 
All charged particles, as well as all photons, traversing the RPCs will therefore interact in the material behind and very close to the RPCs. Most of the particles produced in these interactions will go outwards and be absorbed in the coil material, a small fraction, however, will go backwards and hit the RPCs again.

The existence of back-scattering is demonstrated in Fig. 20. It shows the $z$ coordinate of the
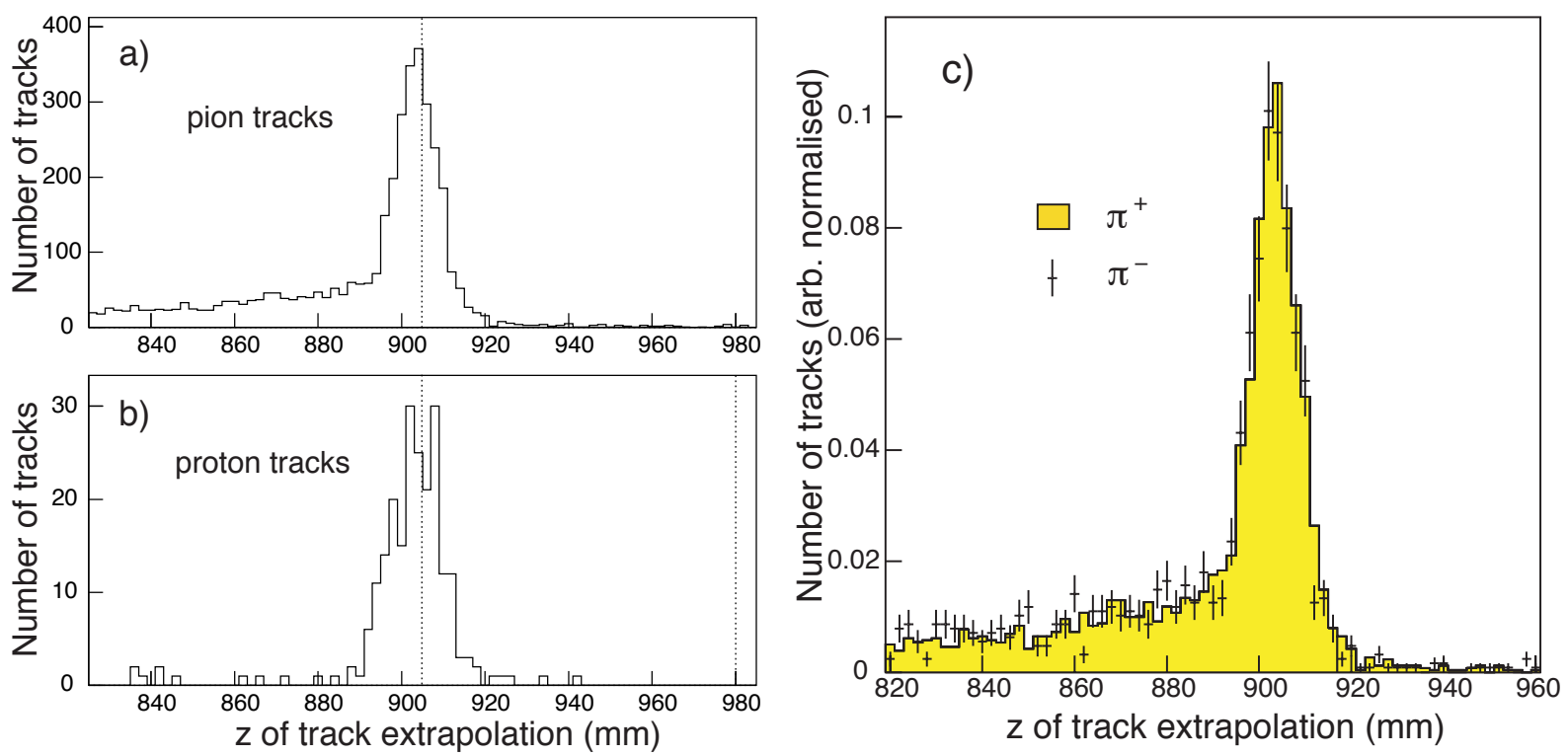

Fig. 20: The $z$ coordinate of the track extrapolation in the TPC for cases where pads 6 and 7 in the same RPC have a hit. The left plot shows the case for pion (a) and proton (b) tracks. The dotted lines indicate the border between pads 6 and 7 . The right plot (c) shows the same for positively and negatively charged pions.

track extrapolation to the RPCs for well-reconstructed charged tracks in the TPC for events were two successive pads in the same RPC had a hit. The plots show the transition region between pads 6 and 7. The peaks at the pad boundaries are generated by tracks which either traverse both pads or pass very close to the neighbour pad such that part of the charge is picked up there. In addition there are tracks which clearly do not point to the pad boundary but, nevertheless, give rise to hits in the neighbour pad (upper left and right plot). While electronics cross-talk would result in a uniform background over the full pad length these tracks are asymmetrically distributed, populating preferentially the pad that is closer to the target. The number of such tracks within a pad increases as one approaches the pad boundary. We attribute the accompanying hits in the neighbour pad to back-scattering. The fraction of tracks with back-scattering is a function of the polar angle under which the track traverses the RPC; it is small for tracks hitting the RPCs more or less under $90^{\circ}$ but reaches values of $15-20 \%$ for tracks hitting pads rings 7 and 8.

Figures 20a and $\mathrm{b}$ show that back-scattering is very different for pion and proton tracks; for protons we almost do not observe back-scattering in the RPCs. Figure 20c compares backscattering induced by positively and negatively charged pions, there is no significant difference.

The charge spectrum of the hits induced by back-scattered particles is compared in Fig. 21 (a) with the charge spectrum for direct hits (b). We have selected tracks pointing to strip 7 in pad 7, i.e. tracks with relatively shallow angles of incidence for which back-scattered particles 
have a high probability to hit pad 8, and required an RPC hit in pad 7 plus an additional RPC hit (without track) in pad 8 . Figure 21 b shows the charge spectrum in pad 7 while Fig. 21a shows the charge spectrum in pad 8 . The shape of the latter suggests that back-scattering is dominated
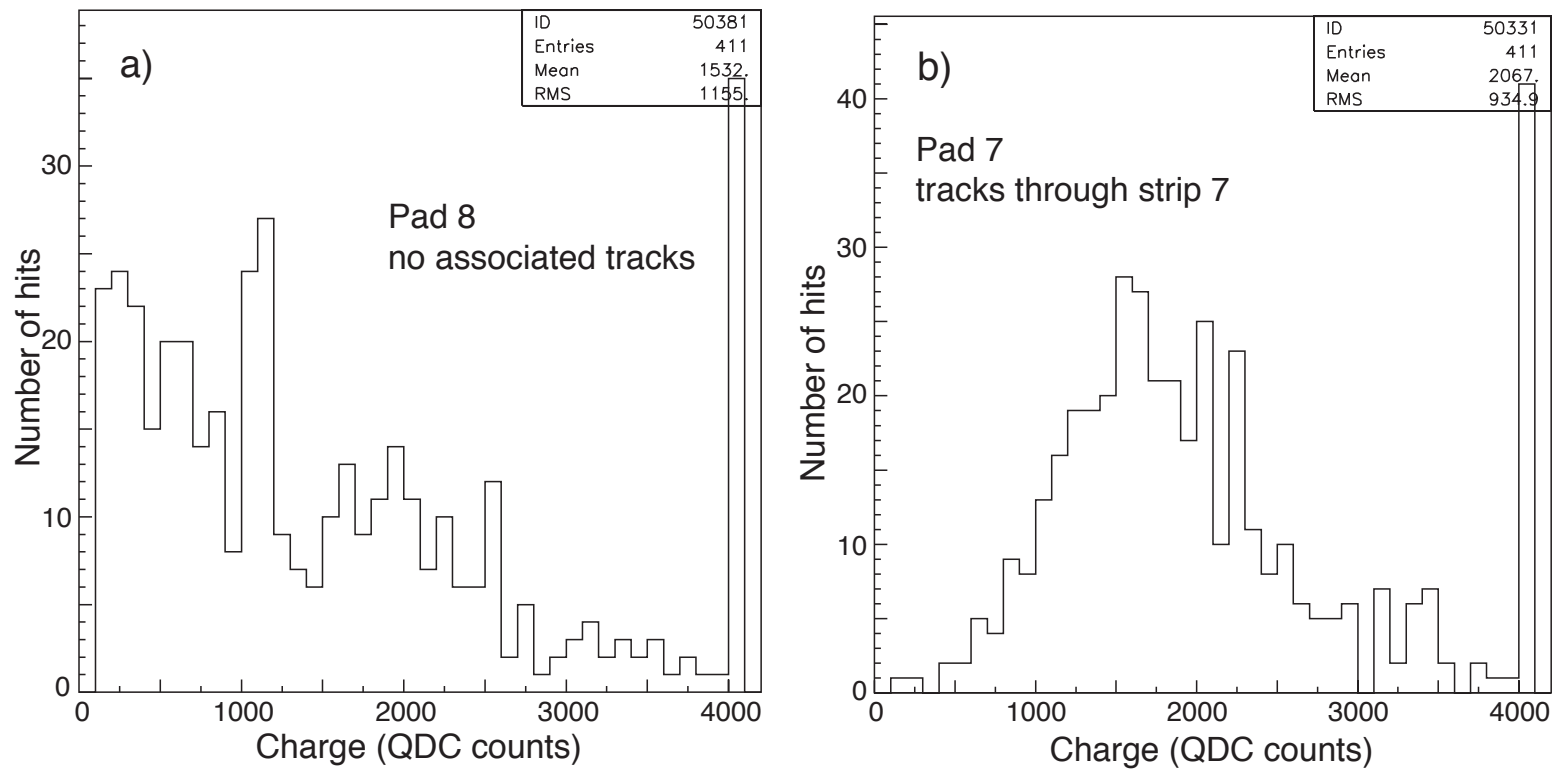

Fig. 21: Charge spectrum of the initial pion track in strip 7 of pad 7 (a) and accompanying charge spectrum in pad 8 (b).

by low-momentum $(\sim \mathrm{MeV} / c)$ photons interacting in the RPCs via Compton scattering $\left.{ }^{20}\right)$. The induced hits occur shortly (order $100 \mathrm{ps)}$ ) after the direct signal, depending on the angle of the initial track and the (unknown) direction of the back-scattered particle. It will not significantly change the time signal but add charge and thus affect the time-slewing correction. From the observed charge spectrum we also cannot exclude a contribution of low-momentum hadrons being back-scattered; those would give rise to very large charges and thus a very quick signal rise but would, on the other hand, be very much delayed before they hit the RPC.

Not knowing on an event-by-event basis whether there was back-scattering, nor its effect on the time measurement, we adopted a pragmatic approach and extracted average corrections. Back-scattering, therefore, leads to a worsening of the time resolution and contributes to the tails of the resolution distributions.

An overall average timing bias because of back-scattering is taken into account automatically for pions in the way pad-specific modifications of the global time-slewing correction and $t_{0}$ 's are determined. For protons a pad-ring-dependent timing bias has been determined and will be discussed in the context of the $t_{0}$ determination in Section 5.2.

\section{$5.2 t_{0}$ calibration}

The $t_{0}$ is a constant which fixes the zero point of the time scale for each individual RPC channel. It depends on unknown electronics transition times and cable delays and must be determined separately for each pad. It is defined through the relation

$$
t_{\mathrm{TOF}}=t_{\mathrm{RPC}}-t_{\mathrm{tgt}}-t_{0}
$$

\footnotetext{
${ }^{20)}$ Electrons from Compton scattering, in most cases, give rise to signals in a single gas gap of the RPC only, before they get absorbed in the glass. For our small gap size, this results in a falling charge spectrum.
} 
where $t_{\mathrm{TOF}}$ is the time-of-flight of the particle, $t_{\mathrm{RPC}}$ is the time measured by the RPCs, after all corrections, and $t_{\text {tgt }}$ is the time of arrival at the target of the beam particle.

We have explored two roads to determine the $t_{0}$ 's, one using 'neutral' hits, the other using charged tracks. We call neutral hits signals in the RPCs without any track in the TPC pointing to the corresponding RPC pad. Neutral hits have the advantage that their timing is generally the one of a photon from $\pi^{0}$ decay in the target, moving along a straight trajectory with speed of light. Their disadvantage lies in the fact that their impact point on the pad is not known, and that their origin from photons from $\pi^{0}$ decays in the target is a good approximation but not certain. We have renounced using neutrals for the precision calibration of the barrel RPCs because a large fraction of the neutral hits in the barrel stems not from conversions of photons in the outer field cage of the TPC but from conversions in the material behind the RPCs. Back-scattered low-energy photons will then induce hits with unknown delays in the RPCs through Compton scattering ${ }^{21)}$.

We retained the method with charged tracks and have used well-reconstructed $\pi^{-}$tracks with $0.25 \mathrm{GeV} / c \leq p_{\mathrm{T}} \leq 0.9 \mathrm{GeV} / c$. We define $t_{0}$ as the most likely value of the time measured by the RPCs, with all its corrections, minus the arrival time of the beam particle at the target minus the expected time of flight for a pion with momentum as reconstructed in the TPC:

$$
t_{0}=t_{\mathrm{RPC}}-t_{\mathrm{tgt}}-t_{\mathrm{TOF}}^{\text {expected }} .
$$

By using charged pions for the determination of the $t_{0}$ constants we effectively include contributions of back-scattering in the calibration. For proton tracks we do not observe backscattering and therefore the pion $t_{0}$ 's will not be entirely correct for them. We estimate the size of the difference in the $t_{0}$ 's of pions and protons related to back-scattering as follows. From an average additional charge of 1000 QDC counts induced by the back-scattered particle one expects a difference of about 200 ps from the time-slewing correction for an average charge of 2000 QDC counts for the initial hit. We estimate that on average $10 \%$ of the pion-induced hits are affected by back-scattering and therefore expect differences between pion and proton $t_{0}$ 's of the order of 20 ps. For small pad ring numbers the expected effect from the time slewing is somewhat larger because of the smaller primary signal charges involved, at the same time the rate of back-scattering is lower than $10 \%$. For high pad ring numbers the rate of back-scattering is larger but the error in the time-slewing correction is smaller.

The long-term behaviour of the $t_{0}$ 's was studied for different target and run settings ${ }^{22)}$. We observed global timing shifts of up to 200 ps between run periods about one month apart, with channel-to-channel variations of $\sigma=50-80 \mathrm{ps}$, probably the result of long-term threshold drifts of the splitter electronics. We therefore use a specific set of $t_{0}$ 's for each data-taking period.

\subsection{System time resolution}

The accuracy of the time-of-flight measurement depends to about $50 \%$ on the intrinsic time resolution of the RPCs. The remaining $50 \%$ stem from the uncertainty in the measurement of the arrival time of the beam particle and the various uncertainties in the corrections applied.

The use of TOF for particle identification rests on a convoluted time resolution, on the one hand the RPC time resolution, on the other hand the time resolution caused by the uncertainty in

\footnotetext{
${ }^{21)}$ In the forward RPCs there is very little material behind the RPCs and we conjecture that the number of additional hits from back-scattered particles is negligible.

${ }^{22)}$ A run setting lasted not more than a few days.
} 
the momentum measurement in the TPC. Figure 22a shows this convoluted resolution function for $\pi^{-}$particles hitting pad ring 7 . The central part of the distribution is well represented by
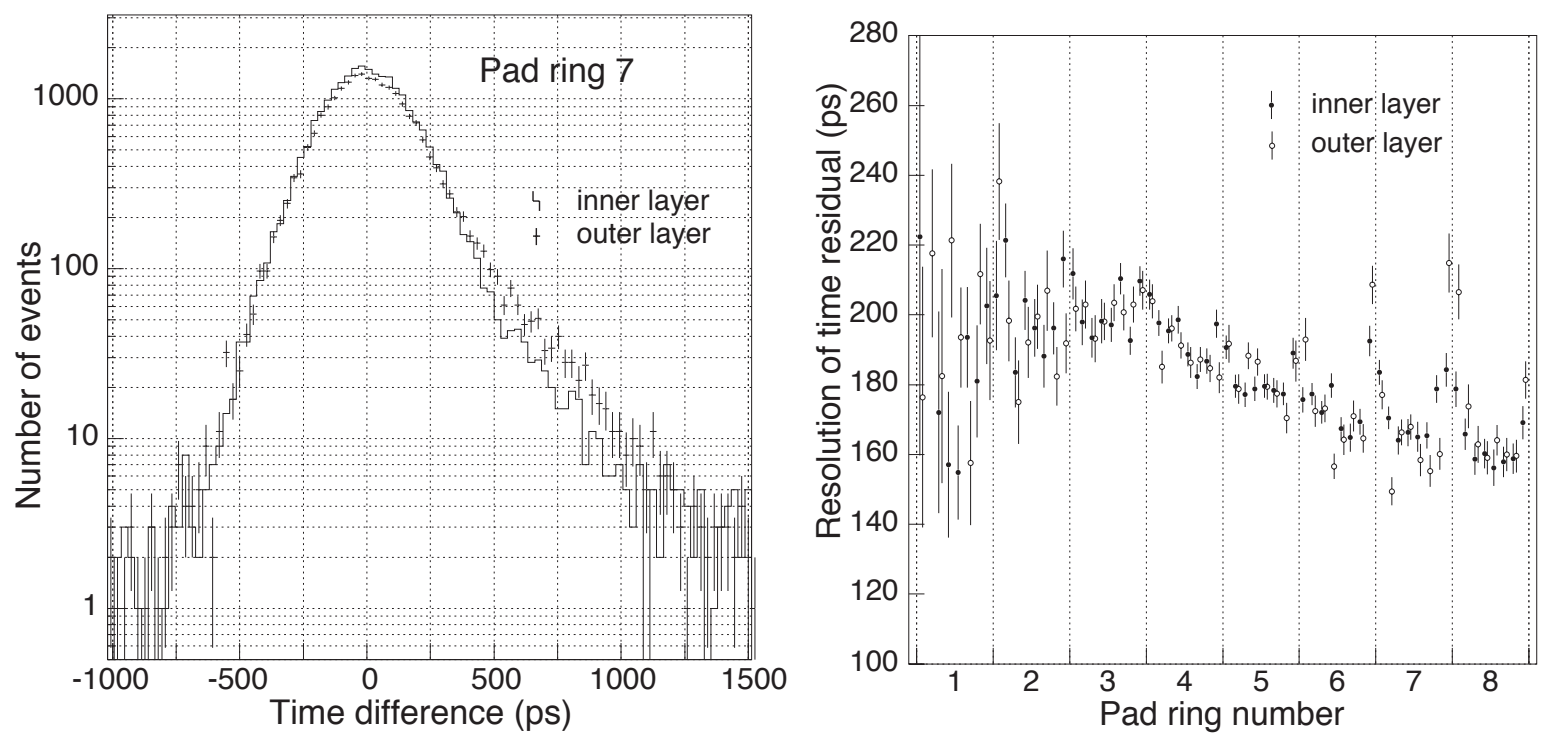

Fig. 22: a) Distribution of differences between TOF as measured in the RPCs and TOF expected from the track fit in the TPC for tracks passing through pad ring 7, separately for the RPCs in the inner and outer layer; the Gaussian standard deviation is 165 ps; b) Sigma of the Gaussians fitted to the time differences in the 64 strip rings of the barrel RPCs, separately for the RPCs in the inner and outer layer.

a Gaussian. The tails towards larger positive time differences are attributed to back-scattering. The convoluted resolution across the eight pad rings, separately for the 64 strips, is shown in Fig. 22b. It varies between $\sim 200$ ps for tracks hitting pad ring $3\left(\sim 90^{\circ}\right)$ and 165 ps for tracks hitting pad rings 7 and 8. It comprises all contributions to the time resolution of the RPCs listed in Table 3 and the contribution from the track reconstruction in the TPC. For pions the latter is a small contribution, but it is important for protons.

Table 3: Contributions [ps] to the overall time-of-flight resolution averaged over the barrel RPCs

\begin{tabular}{|l|c|}
\hline Intrinsic time resolution & 127 \\
Remanent temperature dependence & 20 \\
Different strip delays & 20 \\
Time-slewing correction & 30 \\
Strip transit time & 20 \\
$t_{0}$ constant & 20 \\
\hline Beam particle arrival & 107 \\
\hline \hline RPC system time resolution & 175 \\
\hline
\end{tabular}

\subsection{System efficiency}

While the intrinsic RPC efficiency is close to $100 \%$, the efficiency for observing a hit in the RPCs for a track in the TPC is lower because of the absorption of particles in the structural 
material between TPC and RPCs. We call this 'system efficiency'. It depends on the particle species, its momentum, and the track inclination. Figure 23 shows the loss of momentum for protons (a) and pions (b) in the structural material between the TPC and the RPCs as a function of the particle momentum in the TPC. The momentum loss is largest for pad ring 8 which is hit
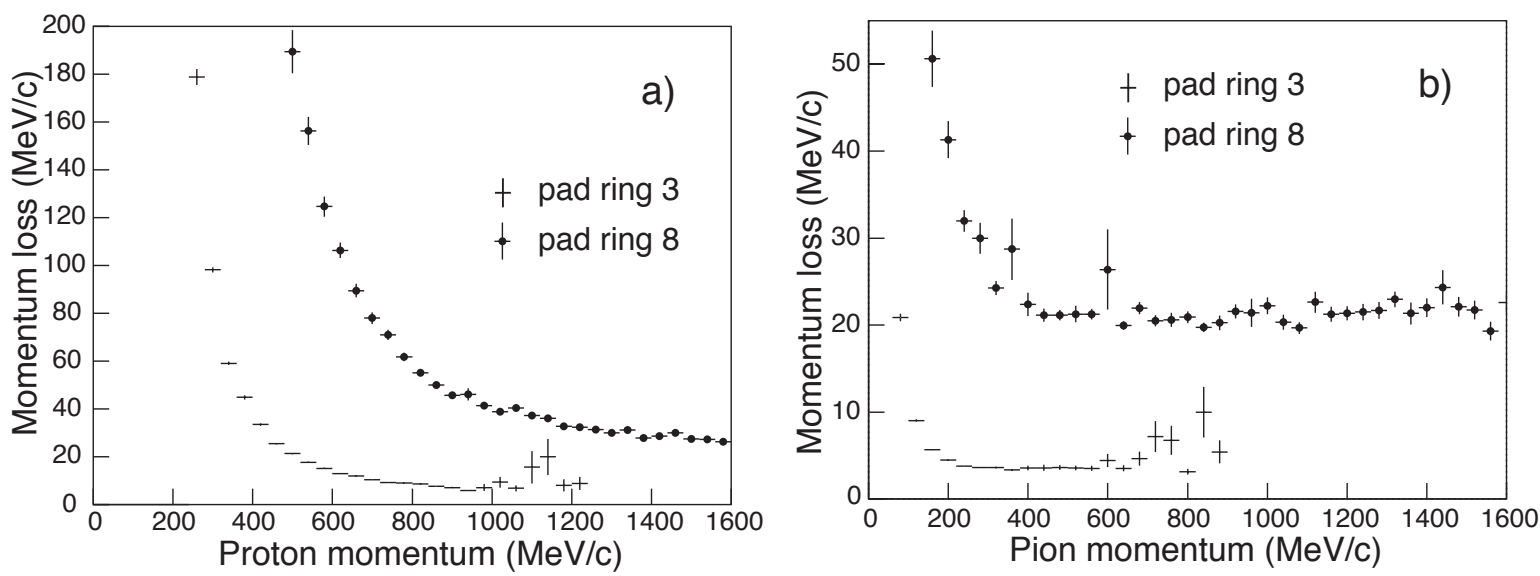

Fig. 23: GEANT4 simulation of the loss of momentum from energy loss in the structural materials between the TPC and the barrel RPCs as a function of the particle momentum as measured in the TPC; shown are the results for pad ring 3 and 8 , representing the best and worst cases.

with an average polar angle of $\sim 19^{\circ}$, and smallest for pad ring 3 which is hit under polar angles close to $90^{\circ}$. There is a cut-off in momentum, below which the particles range out and do not reach the RPCs. For example, protons with momentum less than $250 \mathrm{MeV} / c$ do not reach pad ring 3.

Figure 24a shows the system efficiency for well-reconstructed tracks as a function of their transverse momentum in the TPC for positive and negative pions, averaged over the full barrel RPC system. The tracks have been selected on the basis of their $\mathrm{d} E / \mathrm{d} x$ measurement in the TPC. Figure $24 \mathrm{~b}$ shows the result of a GEANT4 simulation under the assumption of $100 \%$ intrinsic RPC efficiency. The measured efficiencies of $97-98 \%$ are fully consistent with the results obtained in the calibration scan discussed in Section 4.1. The drop in efficiency for $p_{\mathrm{T}}<0.1$ is explained by the increasing energy loss for pions with momenta below $100 \mathrm{MeV} / c$. This is confirmed by the results of the GEANT4 simulation. The simulation predicts slightly smaller efficiencies and also smaller differences between positive and negative pions than observed in the data ${ }^{23)}$. We conclude that the system inefficiency is dominated by the particle absorption in the material between TPC and RPCs.

\subsection{Particle identification}

The RPC system was designed to complement the particle identification capability of the TPC (which uses $\mathrm{d} E / \mathrm{d} x$ to separate pions from protons). In the momentum range $150-250 \mathrm{MeV} / \mathrm{c}$ the energy loss of pions and electrons in the TPC gas is comparable and the $\mathrm{d} E / \mathrm{d} x$ measurement is not able to distinguish between them. The primary goal was to reach a precision in the RPC time-of-flight measurement sufficient to separate electrons and pions.

\footnotetext{
${ }^{23)}$ The small difference in efficiency between $\pi^{-}$and $\pi^{+}$is consistent with the higher inelastic cross-section of $\pi^{-}$for momenta below $500 \mathrm{MeV} / c$ [18].
} 

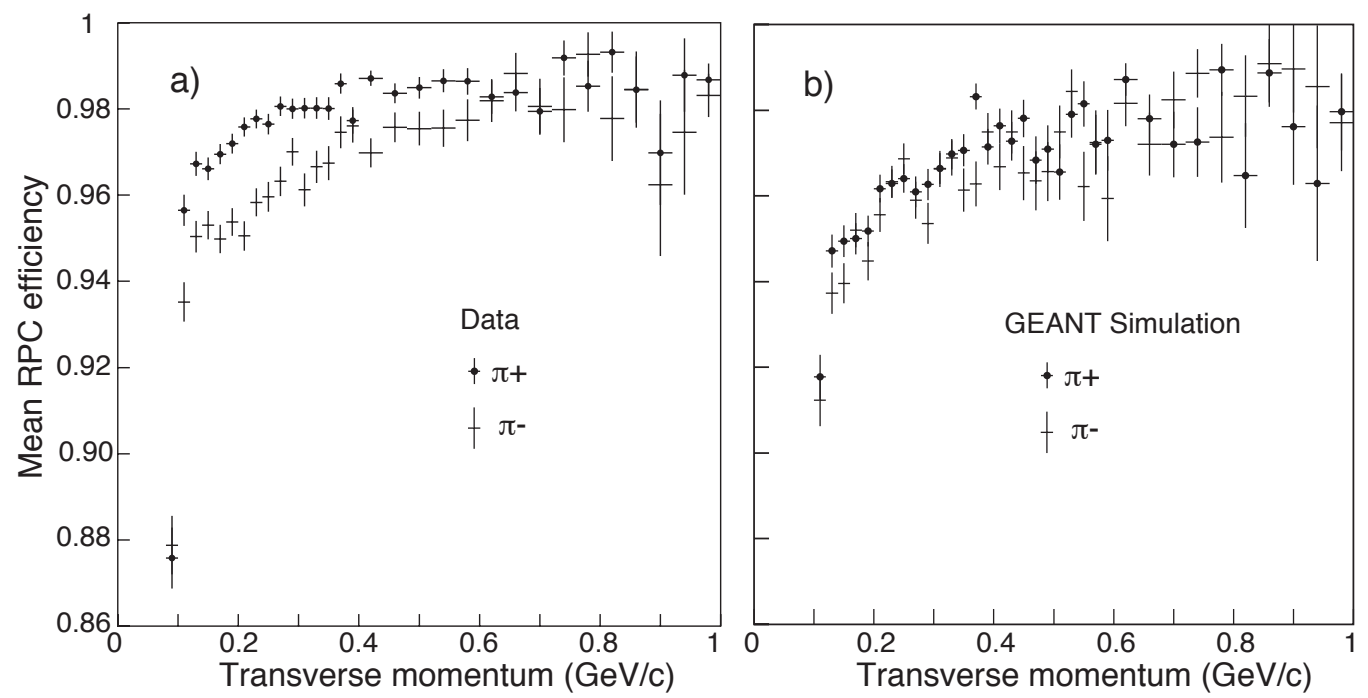

Fig. 24: a) RPC efficiency as a function of transverse momentum for well reconstructed $\pi^{+}$and $\pi^{-}$ tracks. b) GEANT4 simulation, see text.

Figure 25a shows the velocity, as determined by time of flight with the RPCs, plotted against the momentum determined in the TPC, for particles produced in the interactions of $+8.9 \mathrm{GeV} / \mathrm{c}$ hadrons in a $5 \% \lambda_{\text {abs }}$ Be target. The data which extend over the full acceptance of the barrel RPCs follow nearly perfectly the theoretical expectation (a very small offset of the proton band will be discussed below). The all-important capability of separating electrons from pions is evidenced in the right plot which shows this separation up to momenta of $\sim 200 \mathrm{MeV} / c$.

Figure 26a shows the velocity versus momentum for an electron-enriched sample of tracks from photon conversion candidates, where two tracks of opposite sign coming from the same origin and with the same initial direction have been selected. The projection onto the $\beta$ axis giving the resolution in $\beta$ of $7.4 \%$ for the selected sample of electron tracks is shown in Figure 26b. The electron-pion separation capability of the system is even more clearly demonstrated in Fig. 27 where the velocity of track with momenta between 95 and $215 \mathrm{MeV} / \mathrm{c}$ is plotted against the energy loss of the track as measured in the TPC. Electron and pion clusters are well separated in velocity over the full range in which they cannot be distinguished by $\mathrm{d} E / \mathrm{d} x$.

Returning to the offset of the proton band referred to above: it is caused by a combination of the following three effects:

- Energy loss in the detector material: the particle momentum shown in Fig. 25 is the one in the TPC; before entering and after leaving the TPC the particle momentum is different (as a result of energy loss in the target, Inner Trigger Counter, TPC walls, etc.) resulting in a shorter particle time of flight than expected from the measured momentum. For protons the effect (averaged over all polar angles) is $45 \mathrm{ps}$ at $400 \mathrm{MeV} / \mathrm{c}$ and $20 \mathrm{ps}$ at $1200 \mathrm{MeV} / \mathrm{c}$.

- The earlier threshold crossing of the proton signal as discussed in Section 4.9. The size of the effect is $80 \mathrm{ps}$ at $400 \mathrm{MeV} / c$ and $20 \mathrm{ps}$ at $1200 \mathrm{MeV} / \mathrm{c}$.

- The bias of proton timing because of back-scattering, as discussed in Section 5.2, where this effect was estimated to be of the order of $20 \mathrm{ps}$. 

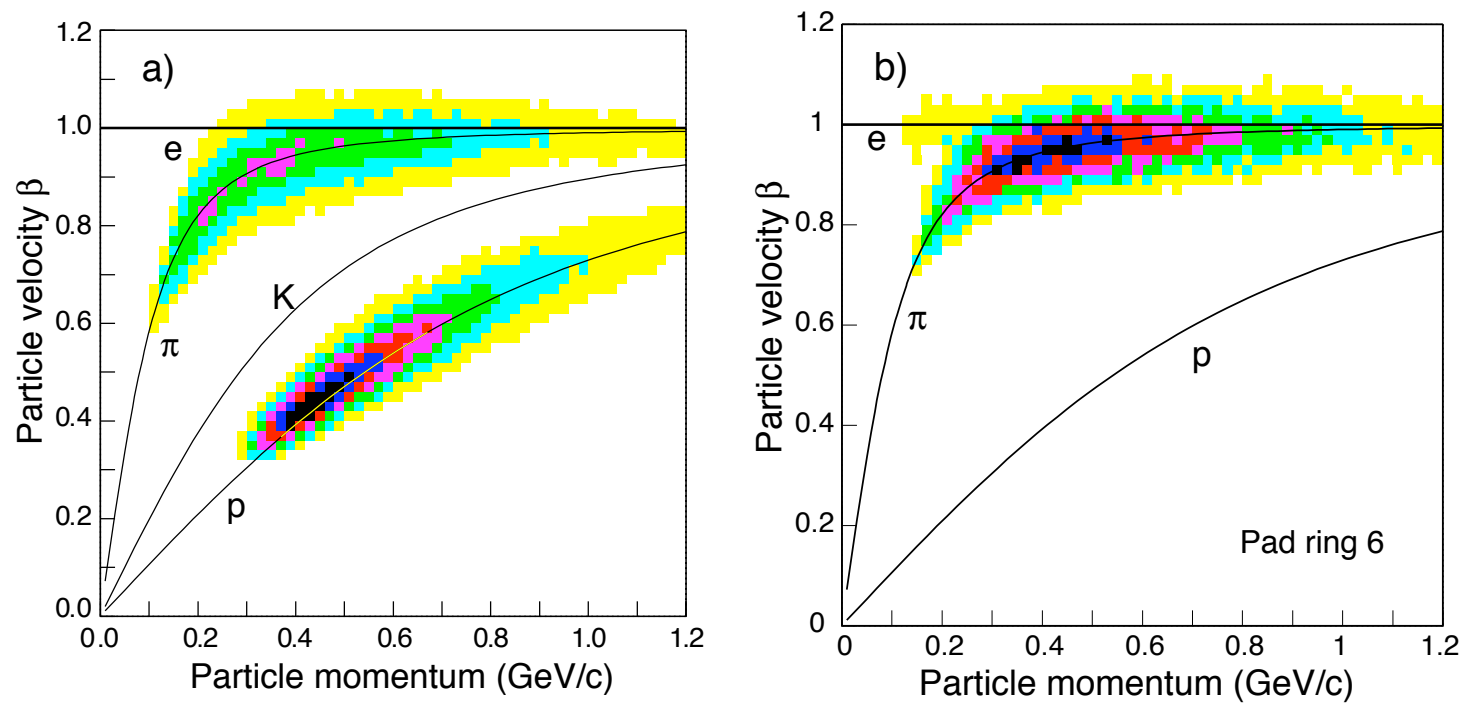

Fig. 25: Velocity versus particle momentum for positive (a) and negative (b) tracks. For positive tracks the full acceptance region of the barrel RPCs is shown, for negatives only tracks hitting pad ring 6 . The lines show the theoretical relations between particle velocity and momentum for electrons, pions, kaons, and protons.
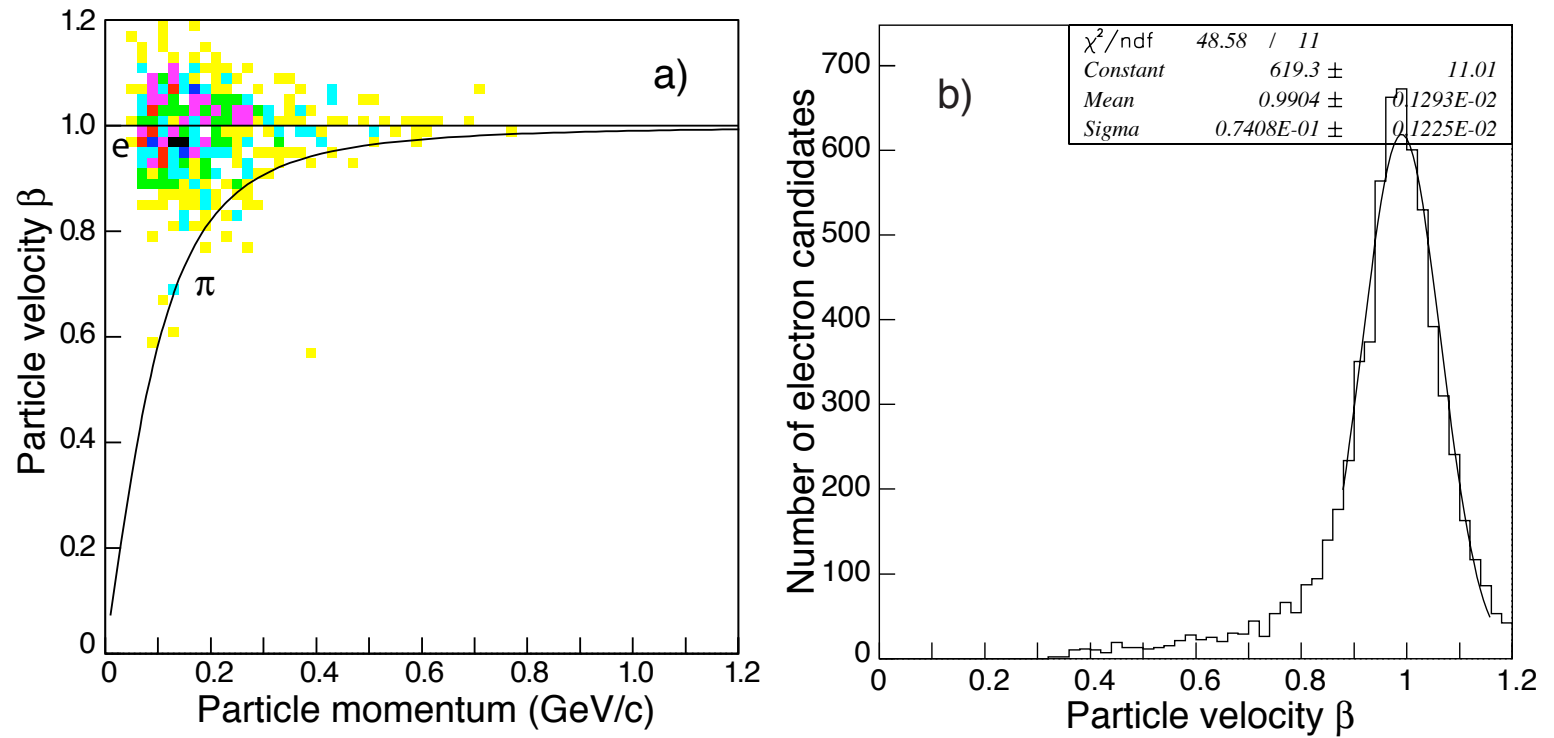

Fig. 26: a) Velocity $(\beta)$ of tracks of an electron-enriched sample of secondary particles as a function of particle momentum as measured in the TPC. b) Projection onto $\beta$ axis showing a resolution in $\beta$ of $7.4 \%$. 

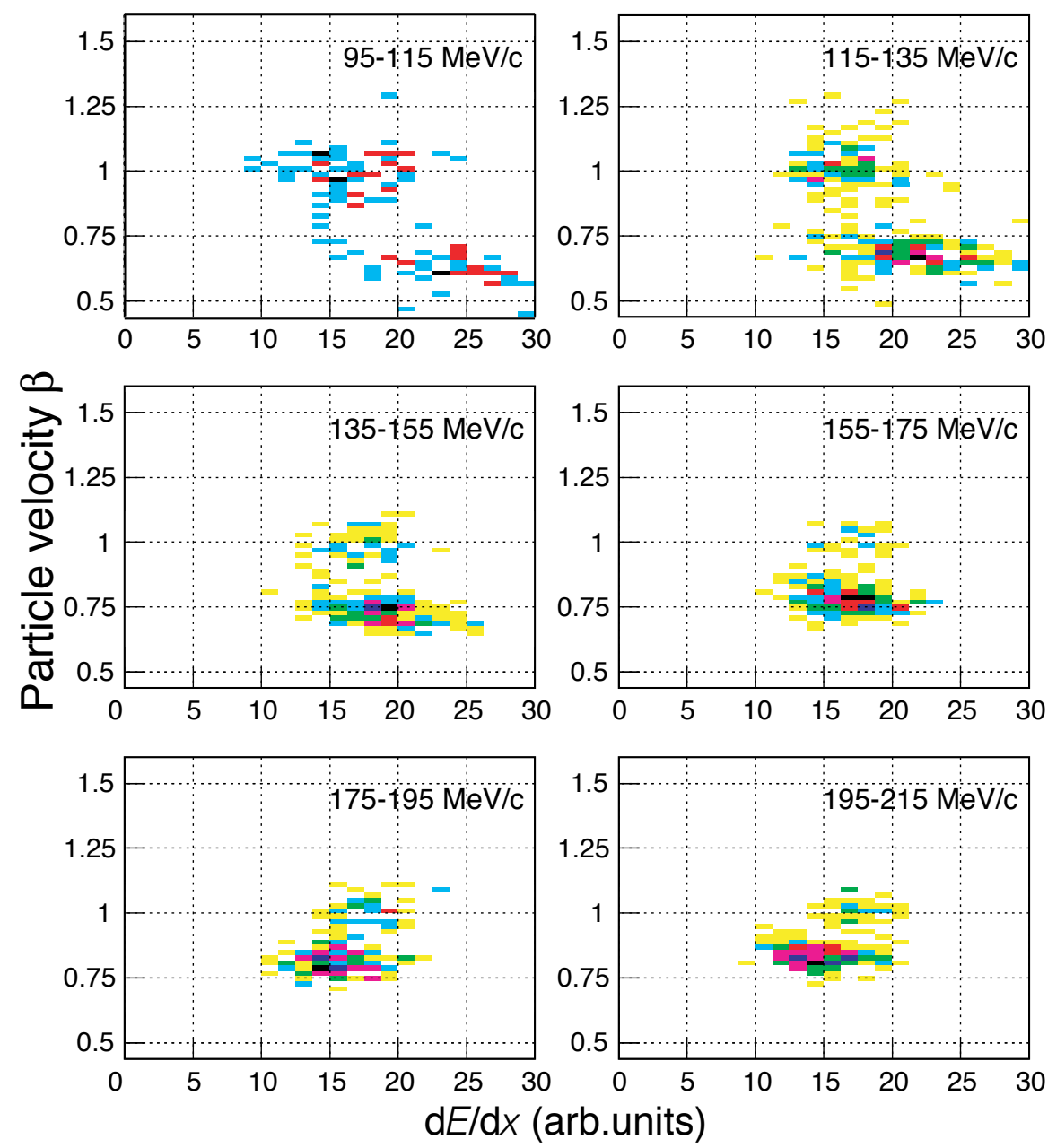

Fig. 27: Normalized velocity of positive secondary particles as measured by the RPCs, for particle momenta in the range $95-215 \mathrm{MeV} / c$, versus their specific ionization $\mathrm{d} E / \mathrm{d} x$ measured in the TPC.

Incidentally all three effects act in the same direction ${ }^{24)}$. Their combined effect amounts to $145 \mathrm{ps}$ for protons with lowest momenta and $60 \mathrm{ps}$ for $1200 \mathrm{MeV} / \mathrm{c}$ protons leading to a 2-3\% upward shift of the proton band with respect to the theoretical curve for low momenta and less than $1-2 \%$ for momenta above $1 \mathrm{GeV} / c$.

\section{SUMMARY}

The design, the operation and the performance for physics analysis of a system of multigap RPCs is described. The salient features are an intrinsic efficiency of almost $100 \%$ and a system efficiency of better than $97 \%$. The intrinsic RPC time resolution is a function of signal charge and reaches values as low as $\sim 100 \mathrm{ps}$. For the time of flight measurement an overall average system time resolution of $\sim 175$ ps has been reached, well matched to the physics aims in the large-angle region of the HARP spectrometer.

\footnotetext{
${ }^{24)}$ In principle there is a fourth effect which acts in the same direction. The most probable value of measured momentum (which is shown in Fig. 25) is smaller than its average value because of the non-Gaussian momentum resolution function. However, for our momentum resolution $\mathrm{d} p_{\mathrm{T}} / p_{\mathrm{T}} \sim 0.3 p_{\mathrm{T}}$ this effect is hardly visible.
} 


\section{ACKNOWLEDGEMENTS}

We are greatly indebted to M.S.C. Williams and his team for competent advice, generous help, and their kind invitation to use for the development of our RPCs the infrastructure of their test facility in the East Hall of CERN's Proton Synchrotron. Warm thanks are due to B. Dybin, A. Golovin, C. Millerin, D. Rotil and Ch. Schillinger for their enthusiasm and devotion to the RPC construction under a very stringent time schedule. We thank M. Bogomilov for his help in the RPC construction and his contributions to the early phase of the analysis. We thank Ch. Wiebusch for the TDC calibration. Further participants in the early analysis were A. Aguilar, G. Barr, A. De Min, A. De Santo, C. Pattison, and S. Robbins whose work was much appreciated. Finally, the authors wish to express their sincere thanks to W. Riegler for instructive and interesting discussions on the theory of RPC timing, and last, but not least, to S. Leech O'Neal for her professional proof-reading.

\section{REFERENCES}

[1] E. Cerron Zeballos et al., Nucl. Instrum. Methods Phys. Res. A374 (1996) 132.

[2] P. Fonte et al., Nucl. Instrum. Methods Phys. Res. A449 (2000) 295.

[3] M. Bogomilov et al., Nucl. Instrum. Methods Phys. Res. A508 (2003) 152.

[4] G. Barr et al., Nucl. Instrum. Methods Phys. Res. A533 (2004) 214.

[5] V. Ammosov et al., Nuclear Physics B (Proc. Suppl.) 158 (2006) 56.

[6] M.G. Catanesi et al., The HARP detector at the CERN PS, Nucl. Instrum. Methods Phys. Res. A571 (2007) 527.

[7] Rebuttal of 'Comments to "The HARP detector at the CERN PS", , by the HARP Collaboration, Nucl. Instrum. Methods Phys. Res. A571 (2007) 564.

[8] M. Bogomilov et al., Physics Performance of the Barrel RPC System of the HARP Experiment, accepted for publication in IEEE Trans. Nucl. Sci.

[9] V. Ammosov et al., Comments on 'The HARP detector at the CERN PS', Nucl. Instrum. Methods Phys. Res. A571 (2007) 562.

[10] V. Ammosov et al., Comment on 'Physics Performance of the Barrel RPC System of the HARP Experiment', submitted to IEEE Trans. Nucl. Sci.

[11] E. Usenko, A fast front-end electronics for the RPC detector in the HARP experiment, HARP Memo 07-102, http://cern.ch/dydak/RPCfrontend.pdf.

[12] M. Shao et al., Meas. Sci. Technol. 17 (2006) 123.

[13] W. Riegler, Ch. Lippmann and R. Veenhof, Nucl. Instrum. Methods Phys. Res. A500 (2003) 144.

[14] W. Legler, Z. Naturforsch. 16a (1961) 253.

[15] S. Ramo, Proc. IRE 27 (1939) 584.

[16] H. Räther, Electron Avalanches and Breakdown in Gases (Butterworth \& Co., London, 1964).

[17] V. Ammosov et al., HARP Memo 06-102, http://cern.ch/dydak/RPCtimingstudy.pdf.

[18] PDG tables, S. Eidelman et al., Phys. Lett. $B 592$ (2004) 1. 